\title{
Simulating the environment around planet-hosting stars
}

\section{Stellar winds and inner astrospheres}

\author{
J. D. Alvarado-Gómez ${ }^{1,2}$, G. A. J. Hussain ${ }^{1,3}$, O. Cohen ${ }^{4}$, J. J. Drake ${ }^{4}$, C. Garraffo ${ }^{4}$, J. Grunhut ${ }^{1}$, and T. I. Gombosi ${ }^{5}$ \\ ${ }^{1}$ European Southern Observatory, Karl-Schwarzschild-Str. 2, 85748 Garching bei München, Germany \\ e-mail: jalvarad@eso.org \\ 2 Universitäts-Sternwarte, Ludwig-Maximilians-Universität München, Scheinerstr. 1, 81679 München, Germany \\ 3 Institut de Recherche en Astrophysique et Planétologie, Université de Toulouse, UPS-OMP, 31400 Toulouse, France \\ ${ }^{4}$ Harvard-Smithsonian Center for Astrophysics, 60 Garden Street, Cambridge, MA 02138, USA \\ 5 Center for Space Environment Modeling, University of Michigan, 2455 Hayward St., Ann Arbor, MI 48109, USA
}

Received 24 May 2016 / Accepted 28 July 2016

\begin{abstract}
We present the results of a comprehensive numerical simulation of the environment around three exoplanet-host stars (HD 1237, HD 22049, and HD 147513). Our simulations consider one of the latest models currently used for space weather studies in the Heliosphere, with turbulent Alfvén wave dissipation as the source of coronal heating and stellar wind acceleration. Large-scale magnetic field maps, recovered with two implementations of the tomographic technique of Zeeman-Doppler imaging, serve to drive steady-state solutions in each system. This paper contains the description of the stellar wind and inner astrosphere, while the coronal structure was discussed in a previous paper. The analysis includes the magneto-hydrodynamical properties of the stellar wind, the associated mass and angular momentum loss rates, as well as the topology of the astrospheric current sheet in each system. A systematic comparison among the considered cases is performed, including two reference solar simulations covering activity minimum and maximum. For HD 1237, we investigate the interactions between the structure of the developed stellar wind, and a possible magnetosphere around the Jupiter-mass planet in this system. We find that the process of particle injection into the planetary atmosphere is dominated by the density distribution rather than the velocity profile of the stellar wind. In this context, we predict a maximum exoplanetary radio emission of $12 \mathrm{mJy}$ at $40 \mathrm{MHz}$ in this system, assuming the crossing of a high-density streamer during periastron passage. Furthermore, in combination with the analysis performed in the first paper of this study, we obtain for the first time a fully simulated mass loss-activity relation. This relation is compared and discussed in the context of the previously proposed observational counterpart, derived from astrospheric detections. Finally, we provide a characterisation of the global 3D properties of the stellar wind of these systems, at the inner edges of their habitable zones.
\end{abstract}

Key words. stars: winds, outflows - stars: mass-loss - stars: magnetic field - stars: late-type - magnetohydrodynamics (MHD)

\section{Introduction}

As well as driving stellar activity cycles, magnetic fields strongly influence different aspects of the stellar structure and evolution. It is known that they play a major role in the coronal heating processes in the Sun and other late type stars (De Moortel \& Browning 2015; Testa et al. 2015), as well as in the generation of persistent stellar winds and astrospheres (see Wood 2004). These stellar winds are crucial to understand the evolution of rotation and magnetic activity in cool stars on the early main sequence. $\mathrm{G}$ to $\mathrm{K}$ type stars tend to rotate rapidly on the zero age main sequence (ZAMS); braking torques exerted by winds cause them to spin down, losing most of their angular momentum within the first 500 Myr (Amard et al. 2016; Barnes \& Kim 2010). Strong winds from the young Sun have been used to explain both the stripping of the Martian atmosphere (Lammer 2013; Terada et al. 2009), and address the "faint young Sun paradox". This paradox is that terrestrial geological records indicate that water existed in liquid form very early in the history of Earth and Mars, despite the young Sun having only $70 \%$ of its current luminosity. Solar wind sputtering is a leading candidate to explain the loss of Mars' once-thick atmosphere, because Mars is not protected by a strong magnetosphere, unlike Earth (see Lundin et al. 2007).
However, recent work presented by Wood et al. (2014) argues that while the young Sun was more magnetically active, it does not necessarily follow that it would have hosted stronger winds.

This last result comes from the close relation between the winds in Sun-like stars and their surrounding astrospheres. In the case of the Sun, the solar wind creates a comet-like bubble (the heliosphere) that extends far past the orbits of the planets, and interacts with the local interstellar medium (LISM) ${ }^{1}$. The heliosphere is populated by hot hydrogen atoms created through charge exchange between the ionized gas in the solar wind and the cold LISM hydrogen. Hot hydrogen builds up particularly in the region between the termination shock and the heliopause. This is the region which the Voyager mission may recently have crossed (Gurnett et al. 2013), although this is still a matter of debate (see Fisk \& Gloeckler 2014; Gloeckler \& Fisk 2015). This hydrogen wall is detected as extra H I Lyman- $\alpha$ absorption in the UV spectra of cool stars. Stronger winds result in a larger astrosphere and increased absorption

\footnotetext{
1 This classical shape of the heliosphere has been recently revisited in various observational and numerical works, pointing towards a far more complex description including magnetized jets (see McComas et al.
} 2013; Opher et al. 2015; Drake et al. 2015). 
(Linsky \& Wood 2014). By measuring the column densities and velocities of this extra absorption it is possible to derive the only available observationally-driven estimates of mass loss rates in cool stars (see Wood et al. 2015). However, these estimates strongly depend on the assumed characteristics and topology of the LISM (see Linsky \& Wood 2014), for which there is still no complete agreement in the literature (e.g., Koutroumpa et al. 2009; Gry \& Jenkins 2014; Redfield \& Linsky 2015).

On the theoretical and modelling side, recent studies have provided different frameworks for the stellar wind origin, behaviour, and influence in the angular momentum evolution of late-type stars. Among the 1D and 2D models, a non-comprehensive list includes semi-empirical approaches for thermally-driven winds, within a hydro- (e.g. Johnstone et al. 2015b, 2015a) or magneto-hydrodynamic (MHD) regime (e.g. Matt et al. 2008, 2012; Réville et al. 2015a,b), physicallymotivated descriptions involving scaling relations for the stellar magnetic fields, rotation periods, convective properties, and X-ray fluxes (e.g. Reiners \& Mohanty 2012; Blackman \& Owen 2016), and semi-analytic and numerical formulations based on Alfvén wave MHD turbulence (e.g. Cranmer \& Saar 2011; Suzuki et al. 2013). While providing reasonable agreement in the rotational evolution of late-type stars at different stages (e.g. Gallet \& Bouvier 2013; Matt et al. 2015), such approaches are very generic and cannot capture the specifics of the stellar wind of a given system. The same is true for the complex interplay between the magnetic field topology, coronal structure, and the stellar wind. These elements are fundamental for a better understanding of the environment around planet-hosting stars, including the relative influence of the wind and the high-energy emission on the exoplanetary conditions and habitability (see Lammer et al. 2003; Lammer 2013; Shaikhislamov et al. 2014; Forget \& Leconte 2014). Such detailed descriptions are crucial for the current and future perspectives in the area of exoplanetary characterisation from the ground and space (see Pepe et al. 2014; Hatzes 2014).

In this context, we presented in Alvarado-Gómez et al. (2016) the initial results of a detailed 3D numerical study aimed at simulating the environment around planet-hosting stars. This previous article described the developed coronal structure and high-energy environment on three exoplanet-hosts, namely HD 22049 (K2V), HD 1237 (G8V), and HD 147513 (G5V). The basic stellar and planetary (orbital) properties in these systems are listed in Table 1.

We employed one of the latest physics-based models, compatible with recent satellite solar observations (see De Pontieu et al. 2007; McIntosh et al. 2011), and currently used for space weather forecast in the solar system (see Tóth et al. 2012). This model considers a data-driven approach where surface magnetic field distributions (i.e. magnetograms) are decomposed in a high-degree spherical harmonics expansion and implemented as an initial condition, following the methodology presented in Tóth et al. (2011). Then the simulation evolves self-consistently, calculating coronal heating and stellar wind acceleration based on Alfvén wave turbulence dissipation (Sokolov et al. 2013; van der Holst et al. 2014).

For other stars this information can be nowadays retrieved (to some extent) from high-resolution spectropolarimetric observations and the technique of Zeeman-Doppler imaging (ZDI, Semel 1989; Brown et al. 1991; Donati \& Brown 1997; Hussain et al. 2000; Piskunov \& Kochukhov 2002). For the stellar systems of interest, ZDI maps were previously recovered by Jeffers et al. (2014), Alvarado-Gómez et al. (2015), and Hussain et al. (2016). As discussed in detail in Alvarado-Gómez et al. (2016),
Table 1. Basic observational properties of the considered systems.

\begin{tabular}{lcccccc}
\hline \hline Star ID & $\begin{array}{c}M_{*} \\
{\left[M_{\odot}\right]}\end{array}$ & $\begin{array}{c}R_{*} \\
{\left[R_{\odot}\right]}\end{array}$ & $\begin{array}{c}P_{\text {rot }} \\
{[\text { days }]}\end{array}$ & $\begin{array}{c}M_{\mathrm{p}} \sin i \\
{\left[M_{\mathrm{J}}\right]}\end{array}$ & $\begin{array}{c}a \\
{[\mathrm{AU}]}\end{array}$ & $e$ \\
\hline HD 1237 $^{a}$ & 0.86 & 1.00 & 7.00 & 3.37 & 0.49 & 0.51 \\
HD 22049, $^{b,}$ & 0.74 & 0.86 & 11.68 & 1.05 & 3.38 & 0.25 \\
HD 147513 & 0.98 & 1.07 & 10.00 & 1.21 & 1.32 & 0.26 \\
\hline
\end{tabular}

References. (a) Naef et al. (2001); Ghezzi et al. (2010); Alvarado-Gómez et al. (2015). ${ }^{(b)}$ Drake \& Smith (1993); Donahue et al. (1996); Valenti \& Fischer (2005); Butler et al. (2006). ${ }^{(c)}$ Mayor et al. (2004), Takeda et al. (2007); Hussain et al. 2016. (†) The listed orbital parameters in Alvarado-Gómez et al. (2016) for the exoplanet in this system were taken from the discovery paper (Hatzes et al. 2000).

we employed two different implementations of this mapping technique (i.e. ZDI and SH-ZDI). The first one considers an image reconstruction with independent pixels for the radial, meridional, and azimuthal components, yielding an unconstrained distribution of the magnetic field (Brown et al. 1991; Donati \& Brown 1997). The second one follows the methodology presented in Hussain et al. (2001) and Donati et al. (2006), where the vector field is reconstructed in terms of a spherical harmonics decomposition. The main difference with the previous approach is the possibility to impose physical and geometrical constraints to the final reconstructed topology (i.e. purely potential or toroidal fields, symmetry or antisymmetry). In this way, the $\mathrm{SH}-$ ZDI implementation permits the completion of the map in the un-observed hemisphere (due to the inclination of the star). As explained in Alvarado-Gómez et al. (2016), the SH-ZDI maps were completed preserving the same level of fit to the spectropolarimetric observations as the ones obtained with the standard ZDI procedure. This last consideration is fundamental due to the fact that the field strengths in the final map depend on the degree of fit to the observations. This ZDI/SH-ZDI comparison was performed in order to evaluate the effect of these observational constraints in our simulations. Additionally, the analysis included a comprehensive evaluation procedure of two solar simulations against satellite data, covering activity minimum (CR 1922) and maximum (CR 1962), that were spatially-filtered to a similar level of resolution as the ZDI maps. Finally, the results of the ZDI-driven stellar simulations were consistently compared with the solar cases, and with various observational estimates of their coronal conditions.

This paper provides continuity to this previous work, to include the stellar wind properties and inner astrospheric structure. A description of the numerical set up, boundary conditions, and general characteristics in each simulation domain is provided in Sect. 2. Section 3 contains the results for each system, including the reference solar cases (i.e., activity minimum and maximum). We discuss our results in the context of previous observational and numerical studies in Sect. 4, and the main conclusions of our work are summarised in Sect. 5.

\section{3D MHD simulation: winds and inner astrospheres}

As in the first paper of this study, the numerical simulations have been performed using the three-dimensional MHD code BATS-R-US (Powell et al. 1999) as part of the Space Weather Modeling Framework (SWMF, Tóth et al. 2005, 2012). We consider the Alfvén Wave Solar Model (AWSoM), which solves the two-temperature MHD equations with additional pressure and energy terms associated with the propagation, reflection, and 
transmission of low-frequency Alfvén waves. The complete description of the code and its numerical implementation can be found in van der Holst et al. (2014).

In this case we analyse the properties of the stellar wind, including the inner region of the stellar corona (SC module) and the resulting structure in the inner astrosphere (IH module). The solar/stellar cases, and the definition of the entire SC component, are identical as in Alvarado-Gómez et al. (2016). This includes base conditions typically assumed in high-resolution solar simulations, to match solar observations such as in-situ wind properties at $1 \mathrm{AU}$ and line-of-sight EUV/X-ray images (Sokolov et al. 2013; Oran et al. 2013). As ZDI reconstructions necessarily have limited spatial resolution because they are insensitive to the small-scale surface field, we used solar magnetograms that have been spatially-filtered to a comparable resolution as the ZDI/SH-ZDI maps. This allows us to better quantify the effects of this limitation in our simulations consistently (see also Alvarado-Gómez et al. 2016). As previously discussed by Garraffo et al. (2013), we expect a much smaller effect in the wind structure than in the X-ray morphology.

The IH component covers the domain from $25 R_{*}$ up to $215 R_{*}$ ( $\sim 1 \mathrm{AU}$ in solar units). The physical conditions in this domain are calculated in the ideal MHD regime. These simulations have been driven by coupling the steady-state stellar corona solutions described in the previous paper of this study, as the inner boundary conditions of the astrospheric component. A $5 R_{*}$ domain-overlap (from $25 R_{*}$ to $30 R_{*}$ ) is used in the coupling procedure between both domains ${ }^{2}$.

For the simulations of HD 1237, the inner astrospheric solution is additionally coupled as a boundary condition of the global magnetosphere $(\mathrm{GM})$ module of the $\mathrm{SWMF}^{3}$. The spatial locations used for this coupling are described in Sect. 3.3. Inside the GM module, the boundary is set at $50 R_{\mathrm{p}}$ (planet radii) towards the central star (day side). The spatial domain for this module extends up to $150 R_{\mathrm{p}}$ in the night side and to $75 R_{\mathrm{p}}$ in the perpendicular directions (orthogonal to the star-planet axis). Both simulation domains (IH and GM components), use a non-uniform cartesian grid which is automatically refined at the location of large gradients either of the magnetic field or plasma density. In this way the geometry of the astrospheric current sheet (in the $\mathrm{IH}$ module), and the bow shock structure (in the GM module) are properly resolved.

The inner boundary condition (located at $\sim 1 R_{\mathrm{p}}$ ), is defined by the planetary parameters of mass, radius, and dipolar magnetic field strength. The mass is taken from the orbital solution determined by Naef et al. (2001). There are no observational constraints for the remaining two parameters. A Jupitersize planet $\left(R_{\mathrm{p}}=R_{\mathrm{J}}\right)$, with a fiducial dipolar field of $B_{\mathrm{p}}=$ $1 \mathrm{G}$, is assumed in this case. Previous observational and numerical studies, focused on hot-Jupiter systems, have suggested stronger planetary magnetic fields (e.g. Shkolnik et al. 2005, 2008; Vidotto et al. 2010, 2012; Llama et al. 2011, 2013). However, the selection of a stronger (weaker) planetary magnetic field would mainly lead to a larger (smaller) size of the magnetosphere, following the relation $R_{\mathrm{M}} \propto B_{\mathrm{p}}^{1 / 3}$ (Eq. (2), Sect. 3.3). As we are interested in characterising the relative effects from the resulting stellar wind structure (and its connection with the

\footnotetext{
2 More details are available in http://csem.engin.umich.edu/ tools/swmf/documentation/HTML/SWMF/index .html

3 See http://ccmc.gsfc.nasa.gov/cgi-bin/display/RT_t. cgi?page=mpause for real-time monitoring of the Earth's magnetosphere, using satellite data and the SWMF.
}

magnetic field topology at the stellar surface), these are independent from the assumed field strength for the exoplanet.

\section{Numerical results}

The results for each simulation regime are presented in the following sections. From the solution in the SC module we computed the mass and angular momentum loss rates (Sect. 3.1). Inside the IH module, the steady-state solution led to the global structure of the wind and associated current sheet (Sect. 3.2). As in Alvarado-Gómez et al. (2016), we perform a consistent comparison between the solar and stellar cases. This includes the solar minimum and maximum activity states, as well as the ZDI/SH-ZDI driven simulations for the stellar cases. Finally, the results of HD 1237 are presented in Sect. 3.3, including the coupled solutions of the GM module, at two critical locations of the exoplanetary orbit.

\subsection{Alfvén surface, mass and angular momentum loss rates}

We initially describe the properties of the solar/stellar wind inside the SC module. Figures 1 to 3 show the equatorial distribution of the plasma density $n$, and radial wind speed $u_{\mathrm{r}}$ (left and middle panels, respectively), extracted from the corresponding steady-state solutions. The right panels contain the distribution of $u_{\mathrm{r}}$ over the current sheet structure, which is defined as the iso-surface with $B_{\mathrm{r}}=0$. We also compute the resulting Alfvén surface (AS) for each solution, displayed as a translucent shade in Figs. 1 to 3. This is performed by calculating the spatial locations at which the Alfvénic Mach number $M_{\mathrm{A}}=u_{\mathrm{sw}} / v_{\mathrm{A}}=1$. In this relation $u_{\mathrm{sw}}$ represents the local stellar wind speed, while $v_{\mathrm{A}}$ is the Alfvén speed of the plasma, determined by the ratio $B / \sqrt{4 \pi \rho}$, with $B$ and $\rho$ as the local magnetic field strength and density, respectively.

Figure 1 shows the results of the solar simulations in this domain. For both activity states, the expected global thermodynamical properties of the solar wind are achieved; relatively fast and low density during solar minimum, and considerably slower and denser for activity maximum. Additionally, as evidenced by the developed current sheet (right panel in Fig. 1) and the geometry of the AS, the overall complexity of the driving magnetic field distribution is reflected in the wind solution. During activity minimum, the current sheet is mostly confined to the equatorial plane (with small deviations in particular sectors of the structure). The AS shows a two-lobe structure aligned with the rotation axis of the star (z-axis), which is usually obtained for simple (nearly dipolar) surface magnetic field distributions (e.g. Vidotto et al. 2014; Cohen \& Drake 2014). For activity maximum, the structure of the current sheet shows warped sectors and greatly departs from the equatorial plane. Similarly the resulting AS in this case shows multiple lobes of irregular sizes, without any preferred orientation in the 3D domain. These fundamental differences are clearly seen in the resulting structure of the inner heliosphere, presented in Sect. 3.2.

The simulations of HD 22049 and HD 1237 led to similar wind structures in the SC domain (Figs. 2 and 3, respectively). Streamers can be observed on the equatorial plane (three for HD 22049 and two for HD 1237), with a mean density $n \simeq 10^{3} \mathrm{~cm}^{-3}$ and radial speeds of $u_{\mathrm{r}} \sim 500 \mathrm{~km} \mathrm{~s}^{-1}$ in the ZDI-driven cases. For the SH-ZDI simulation of HD 22049 two of these streamers are merged, creating a broader high-density sector on the equatorial plane. For both stars, the density drops by a factor of $\gtrsim 100$ between the streamers, while the velocity 

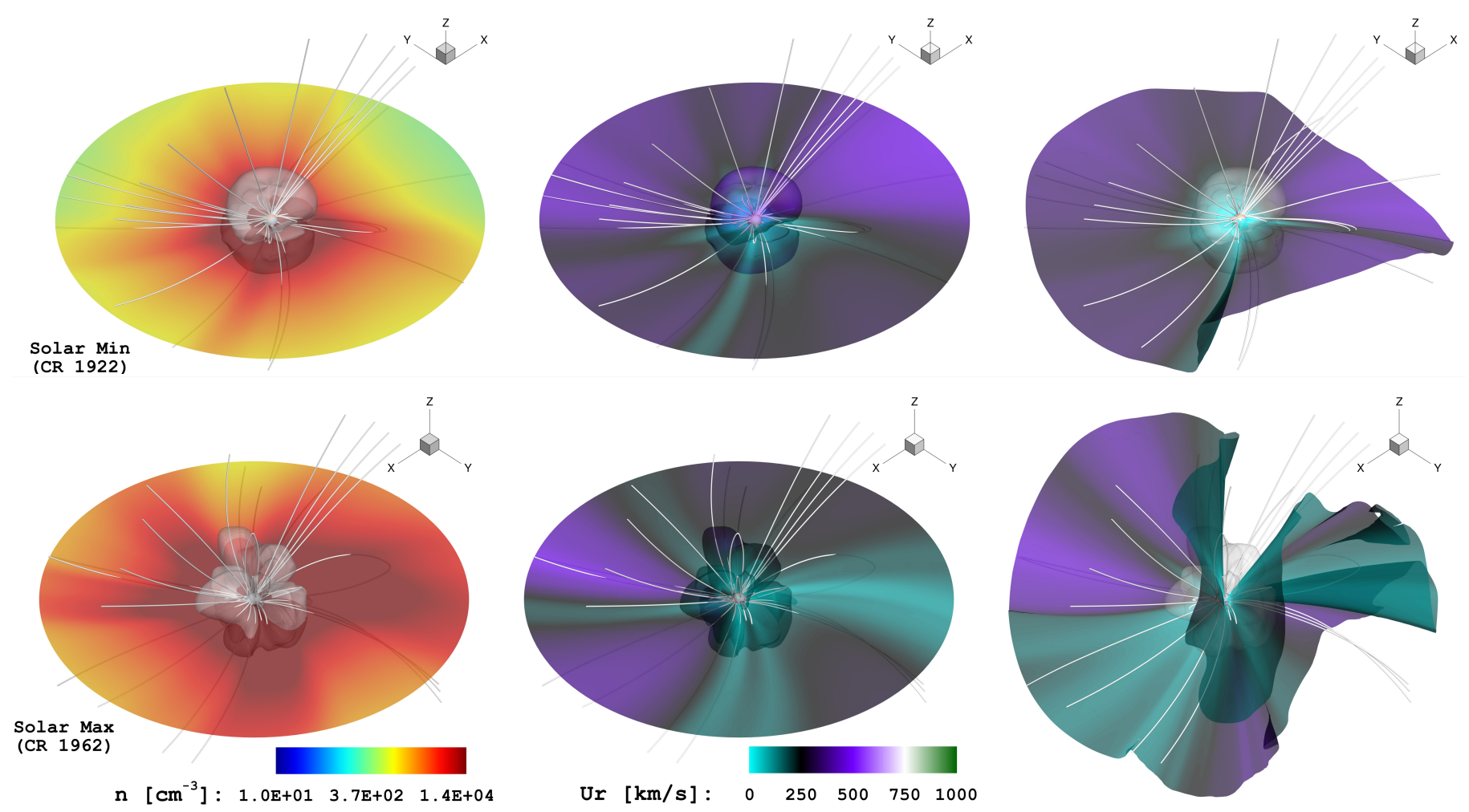

Fig. 1. Simulation results in the solar corona (SC) domain for activity minimum (CR 1922, up) and maximum (CR 1962, down). The left and middle panels contain the projection onto the equatorial plane $(z=0)$ of the plasma density $n$, and the radial wind speed $u_{\mathrm{r}}$, respectively. In the right panel the distribution of $u_{\mathrm{r}}$ on the developed current sheet structure $\left(B_{\mathrm{r}}=0\right)$ is presented. The translucent shade denotes the Alfvén surface $\left(M_{\mathrm{A}}=1\right)$ calculated from the steady-state solution. The corresponding colour scales for $n$ and $u_{\mathrm{r}}$ are preserved among the different panels. Selected 3D magnetic field lines are shown in white.

rises to $u_{\mathrm{r}} \sim 1000 \mathrm{~km} \mathrm{~s}^{-1}$ and $750 \mathrm{~km} \mathrm{~s}^{-1}$ for the ZDI and the SHZDI simulations, respectively. Similarly, a two-lobe AS structure is developed in all the simulations, which is consistently larger in the SH-ZDI cases (see Table 2). The alignment of the AS lobes deviates significantly from the rotation axis, leading to a current sheet structure nearly confined to a plane, highly inclined with respect to the projected stellar equator (Figs. 2 and 3, right panels). The velocity of the wind along the current sheet is approximately $500 \mathrm{~km} \mathrm{~s}^{-1}$ in all cases, with variations up to $\pm 40 \%$ in very small locations of the structure. These structures show a higher density $\left(n \simeq 10^{4} \mathrm{~cm}^{-3}\right)$ in the SH-ZDI simulations, with roughly the same velocity as in the ZDI cases.

Compared to the previously described stellar cases, HD 147513 showed a rather different wind structure in this domain (Fig. 4). The high-density structures of the wind are much wider in this case, with associated radial speeds of $u_{\mathrm{r}} \sim$ $250 \mathrm{~km} \mathrm{~s}^{-1}$. The velocity of the wind remains below $750 \mathrm{~km} \mathrm{~s}^{-1}$ in the equatorial plane, and barely reaches this value in few locations of the 3D domain. Similar to the solar maximum case, the current sheet structure of HD 147513 shows warped sectors and clearly deviates from a planar structure (right panel of Fig. 4). This additional complexity can be also seen in the irregular lobes developed in the AS, which are also common with the solar simulation during activity maximum (Fig. 1, bottom).

These results clearly show the importance of the AS properties on the resulting wind structure. By definition, the AS corresponds to the boundary between magnetically-coupled outflows $\left(M_{\mathrm{A}}<1\right)$ and the escaping stellar wind which no longer exerts torque on the $\operatorname{star}\left(M_{\mathrm{A}}>1\right)$. For this reason it is commonly used in modelling studies to calculate the mass loss rate, $\dot{M}$, and the angular momentum loss rate, $\dot{J}$, associated with the stellar wind (e.g. Cohen et al. 2010; Cohen \& Drake 2014; Garraffo et al. 2015b). Furthermore, as illustrated in Figs. 1 to 3, the topology of the AS reflects to some extent the complexity of the magnetic field distribution driving the simulation (see also Vidotto et al. 2014, 2015; Garraffo et al. 2015a), which in turn, in a self-consistent model, should be directly related to the resulting coronal structure (see Alvarado-Gómez et al. 2016). Finally, previous studies of planet-hosting stars with close-in exoplanets have also shown the importance of the exoplanet location with respect to the AS, which can lead to strong magnetic interactions and angular momentum transfer between the star and the planet (Cohen et al. 2014; Strugarek et al. 2014, 2015).

In addition, the AS provides a common framework to consistently compare our simulations in this domain, and to place our results in context with other studies in the literature. Table 2 contains a summary of the resulting stellar wind properties averaged over the AS, as well as the $\dot{M}$ and $\dot{J}$ values in each case. Several important results are obtained from this quantitative analysis. First of all, by taking an average of the activity minimum and maximum cases, we obtain a mean solar mass loss rate $\left\langle\dot{M}_{\odot}\right\rangle=3.78 \times 10^{-14} M_{\odot} \mathrm{yr}^{-1}$, consistent with the nominal accepted value of $\dot{M}_{\odot} \simeq 2 \times 10^{-14} M_{\odot} \mathrm{yr}^{-1}($ Wood 2004 and references therein), and the observed scatter during the course of the activity cycle (by a factor of $\sim 2$, Cohen 2011).

If we analyse the solar simulations independently, the predicted mass loss rate during activity minimum agrees well with Voyager II data, averaged over the corresponding period of time at the spacecraft (i.e. $\sim 9$ months after $^{4}$ the CR 1922 of

4 Approximate time required for the solar wind to reach Voyager II location, at the average speed predicted by the model in this epoch. 

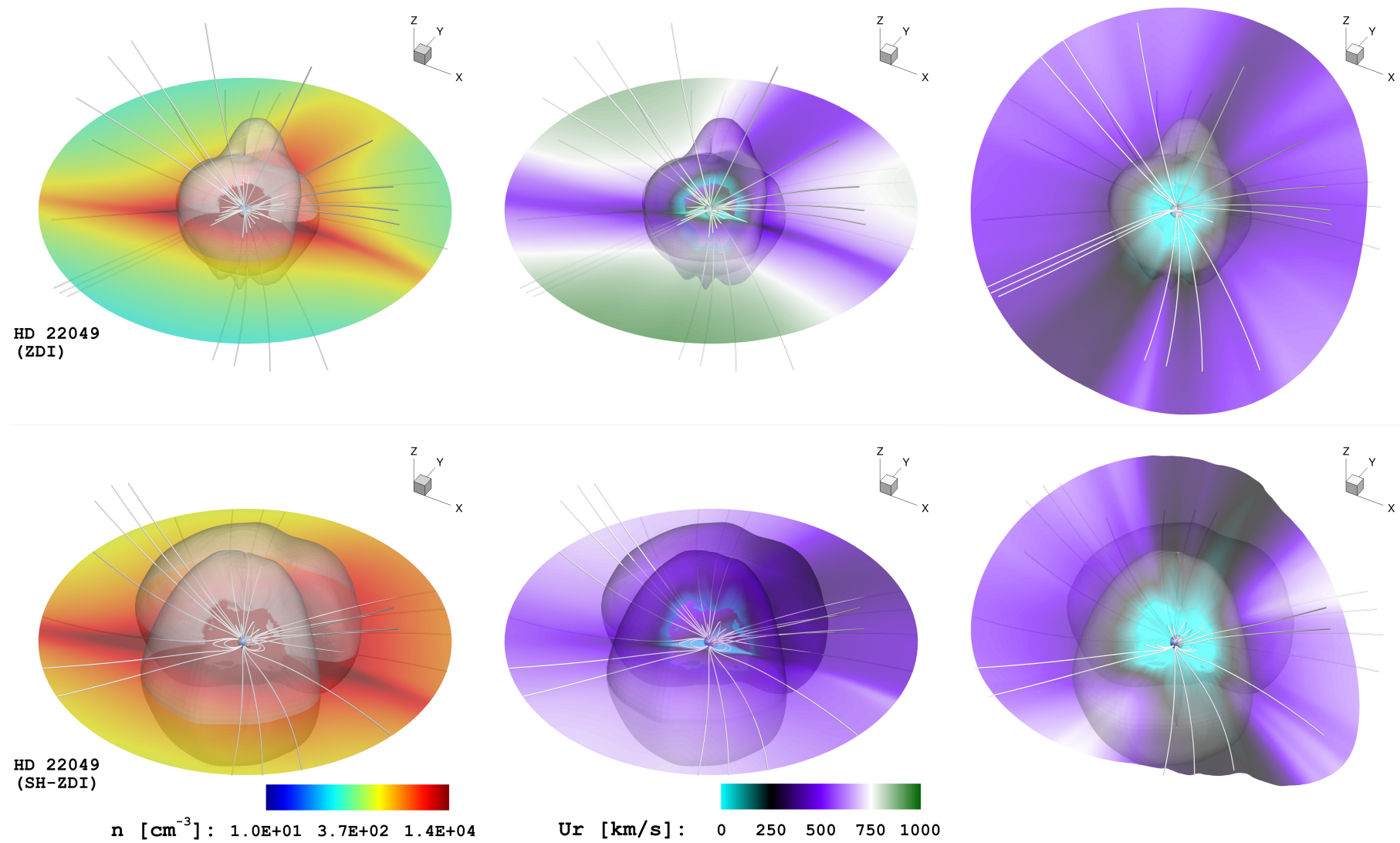

Fig. 2. Simulation results in the SC domain for HD 22049 driven by the ZDI (up) and SH-ZDI (down) magnetic field maps. The left and middle panels contain the projection onto the equatorial plane $(z=0)$ of the plasma density $n$, and the radial wind speed $u_{\mathrm{r}}$, respectively. In the right panel the distribution of $u_{\mathrm{r}}$ on the developed current sheet structure $\left(B_{\mathrm{r}}=0\right)$ is presented. The translucent shade denotes the Alfvén surface $\left(M_{\mathrm{A}}=1\right)$ calculated from the steady-state solution. The corresponding colour scales for $n$ and $u_{\mathrm{r}}$ are preserved among the different panels. Selected 3D magnetic field lines are shown in white.

May 1997). However, a similar comparison for the considered activity maximum epoch (i.e. $\sim 14$ months after the CR 1962 of Apr.-May 2000), indicates that the solar mass loss rate in this case is overestimated by $\sim 40 \%$ (Cohen 2011). This additional mass escaping the star can be interpreted as a deficit of confining loops in the lower corona (inside the AS), which results from the (spatial) resolution-limited magnetograms driving the simulation (see Alvarado-Gómez et al. 2016). While this condition was common among both solar simulations, the effect on the activity maximum case is larger, given the relative amount of complexity and magnetic flux lost in the process of spatially degrading the surface field distribution.

In a similar manner, the simulations yield an average solar angular momentum loss $\left\langle\dot{J}_{\odot}\right\rangle=7.5 \times 10^{29} \mathrm{erg}$. Unlike the observational estimates of $\dot{M}_{\odot}$, values of $\dot{J}_{\odot}$ are more uncertain (ranging between $\sim 10^{29}-10^{31} \mathrm{erg}$ ), and usually determined via numerical models with different assumptions (e.g. Cohen et al. 2010; Matt et al. 2012; Cohen \& Drake 2014; Garraffo et al. 2015b). The relatively small $\left\langle\dot{J}_{\odot}\right\rangle$ resulting from our simulations reflects the average size of the AS directly, which is known to increase with the field strength and decrease with the field complexity (see Réville et al. 2015a; Garraffo et al. 2015a). In the considered solar cases these dependencies are partially compensated, leading to a similar value of $\left\langle R_{\mathrm{AS}}\right\rangle$ in both simulations (see Table 2).

Our result for $\left\langle R_{\mathrm{AS}}\right\rangle$ in the solar minimum case, is very similar to the value obtained by the 2.5-dimensional simulations of Réville et al. (2015a) using the MHD PLUTO code (Mignone et al. 2007) and a similar activity epoch. Interestingly, for activity maximum we obtain a $\left\langle R_{\mathrm{AS}}\right\rangle$ value which is roughly twice compared to their findings. This difference could be related (among with other possibilities), with the dimensional reduction of their approach, with specific properties of the driving magnetic field distribution (i.e. Carrington rotation and instrument used to map the magnetic field), and with the amount of small scale field included in each numerical implementation.

Unlike the average size of the AS, the fundamental properties of solar wind at this region show large variations between both activity states; differences by a factor of $\sim 6$ in the mean plasma density $\langle n\rangle_{\mathrm{AS}}$, and by a factor of $\sim 2$ for the average radial wind speed $\left\langle u_{\mathrm{r}}\right\rangle_{\mathrm{AS}}$. Smaller differences are obtained for the remaining solar wind parameters.

For the considered stellar systems, important differences arise between the ZDI and SH-ZDI cases. For the mass and angular momentum loss rates, the resulting values of $\dot{M}$ and $\dot{J}$ of HD 1237 differ by a factors of $\sim 3$ and $\sim 9$ respectively, being larger in the SH-ZDI case. Similarly, the SH-ZDI simulation of HD 22049 yields $\dot{M}$ and $\dot{J}$ values which are several times larger than in the corresponding ZDI case (i.e. by factors of $\sim 4$ and $\sim 11$, respectively). Smaller differences arise in the average size of the AS, $\left\langle R_{\mathrm{AS}}\right\rangle$, being roughly 1.5 times larger in the SH-ZDI simulations of these two systems. The obtained differences in $\dot{J}$ appear mainly as a result of its direct dependancy with $\dot{M}$ (see Garraffo et al. 2015b). On the other hand, the mass loss rate variations are connected to the relative differences in the surface field distributions driving the simulations (see Alvarado-Gómez et al. 2016), and the Alfvén wave energy transfer to the corona 

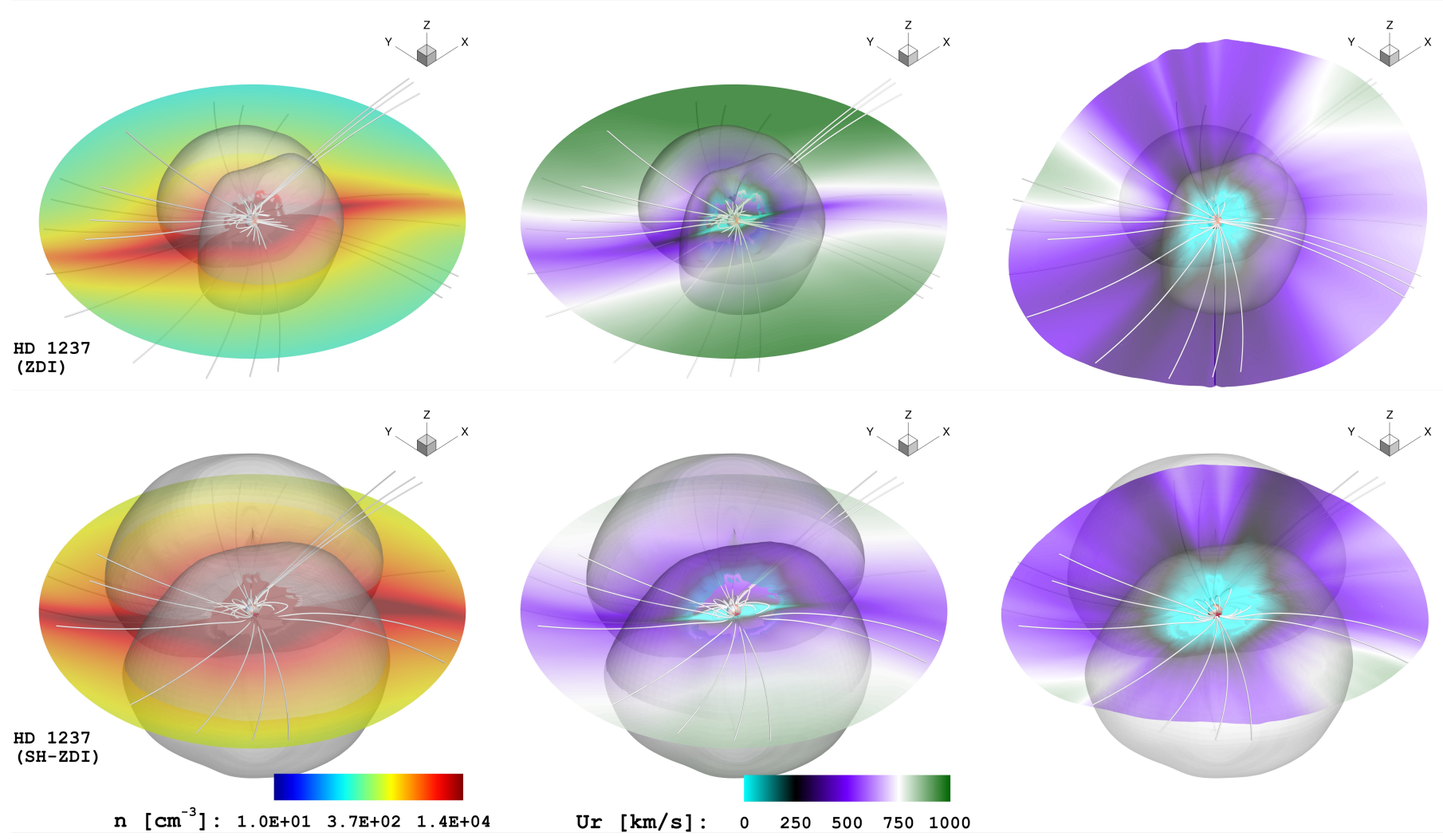

Fig. 3. Simulation results in the SC domain for HD 1237 driven by the ZDI (up) and SH-ZDI (down) magnetic field maps. The left and middle panels contain the projection onto the equatorial plane $(z=0)$ of the plasma density $n$, and the radial wind speed $u_{\mathrm{r}}$, respectively. In the right panel the distribution of $u_{\mathrm{r}}$ on the developed current sheet structure $\left(B_{\mathrm{r}}=0\right)$ is presented. The translucent shade denotes the Alfvén surface $\left(M_{\mathrm{A}}=1\right)$ calculated from the steady-state solution. The corresponding colour scales for $n$ and $u_{\mathrm{r}}$ are preserved among the different panels. Selected 3D magnetic field lines are shown in white.
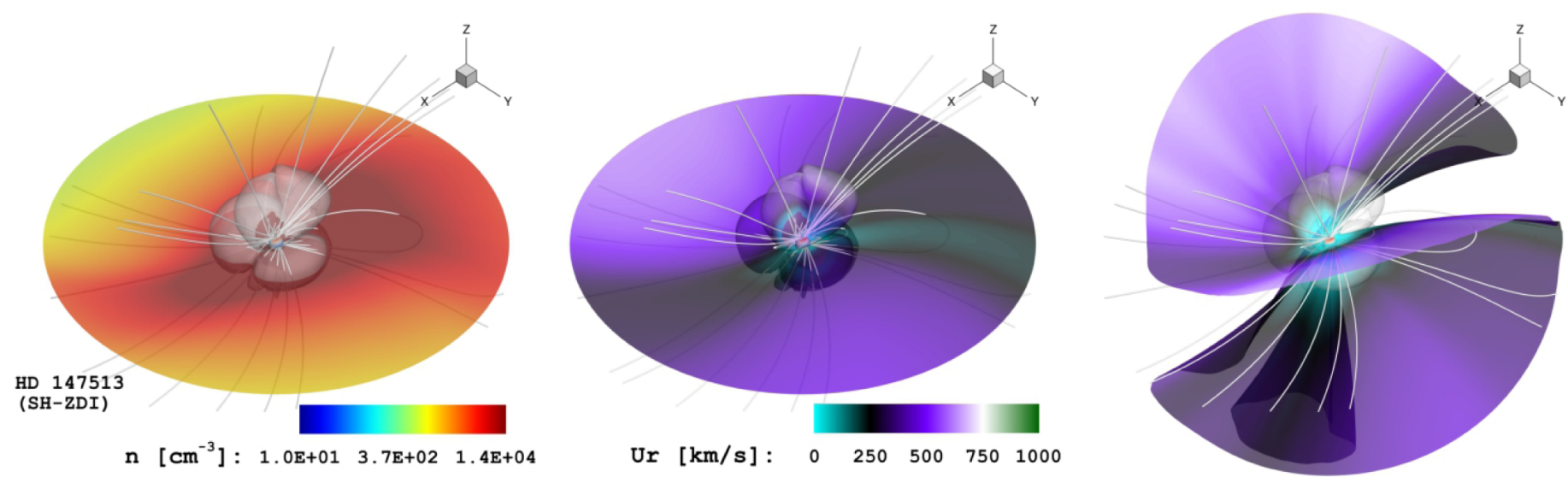

Fig. 4. Simulation results in the SC domain for HD 147513 driven by the SH-ZDI magnetic field map. See caption of Fig. 3.

and wind implemented in the model. The latter is described via the Poynting flux of the emerging Alfvén waves, $S_{\mathrm{A}}$, taken to be proportional to the field strength (and polarity) at the inner boundary of the simulation (i.e., $S_{\mathrm{A}} \propto B_{\mathrm{r}}$; see van der Holst et al. 2014). Previous studies based on the same MHD solver but with a different wind model (i.e., a thermally-driven polytropic stellar wind), do not display significant variations in $\dot{M}$ when considering changes in the magnetic field geometry and/or the incompleteness of the ZDI maps (see Vidotto et al. 2012, which is the basis for the models presented in Vidotto et al. 2014, 2015; do Nascimento et al. 2016; and Nicholson et al. 2016). As the wind-driving mechanism in our simulations is based on Alfvénwave turbulence dissipation, we find much stronger differences in these wind properties based on the large-scale magnetic field geometry. This is indicative of a radical difference between these other models and our simulations.

The remaining stellar wind properties (averaged over the AS), showed less variation between the ZDI and the SH-ZDI cases (see Table 2). Assuming the same initial base conditions, the SH-ZDI simulations led to denser (by $\sim 10-30 \%$ ) and colder (by $\sim 25-35 \%$ ) winds compared to the ZDI-driven cases. As with the solar simulations, the average stellar wind speed at the AS 

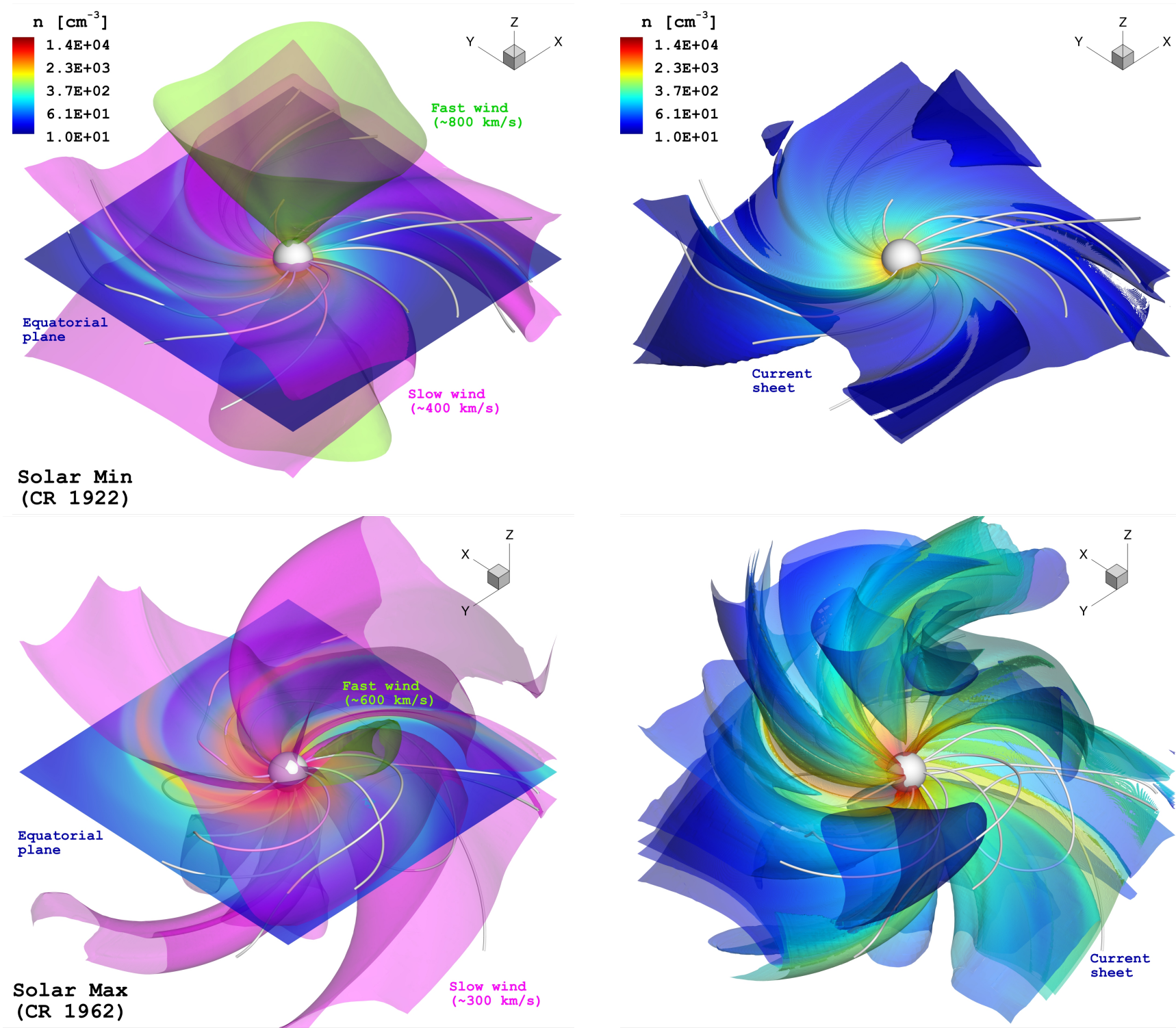

Fig. 5. Simulation results in the inner heliosphere (IH) domain for activity minimum (CR 1922, top) and maximum (CR 1962, bottom). The central white sphere denotes the boundary with the solar corona (SC) domain at $25 R_{\odot}$ (Sect. 3.1). The density structure of the steady-state solution is displayed on the equatorial plane (left) and the heliospheric current sheet (right). In the left panel, the topology and associated magnitudes of the dominant radial velocity components $\left(u_{\mathrm{r}}\right)$ of the solar wind are also included (fast: green - slow: magenta). The density $(n)$ colour scale is preserved among the different panels. Selected 3D magnetic field lines are shown in white.

seems to be lower for higher surface field strengths. These differences are related to the radial behaviour of the thermodynamical quantities (i.e. $\left\langle R_{\mathrm{AS}}\right\rangle$ is larger in the SH-ZDI case), and the underlying coronal structure, which in turn depends on additional factors such as the ZDI map resolution, completeness, and field complexity (see Alvarado-Gómez et al. 2016). This clearly shows the importance of numerical models which selfconsistently simulate both, the corona and stellar wind domains. In the following section, we present the resulting solar and stellar wind properties inside the IH module.

\subsection{Stellar winds and astrospheric current sheet}

As mentioned in Sect. 2, the simulations on the IH domain are driven by the steady-state solutions of the SC region, coupled at $25 R_{*}$ (white sphere in Figs. 5 to 10 and Fig. 12). Similarly to the SC domain, we present the density structure of the wind, projected onto the equatorial plane, and the heliospheric/astrospheric current sheet. The associated colour scale for $n$ is preserved between the SC and IH results, showing the consistency of the coupled MHD solution. Additionally, the 3D structure of the radial wind velocity is visualised via two isovelocity surfaces (translucent shades in Figs. 5 to 9, left), labeled as fast (green) and slow (magenta) wind components. The magnitude of the fast component is calculated using the peak wind velocity achieved in the simulation, and taking the floor with respect to a $100 \mathrm{~km} \mathrm{~s}^{-1}$ velocity bin width. The magnitude of the slow wind component is simply taken as half of the previously defined fast wind. As an example, the solar minimum simulation showed a peak wind velocity of $u_{\mathrm{r}} \sim 885 \mathrm{~km} \mathrm{~s}^{-1}$, so the fast and slow wind iso-surfaces were taken at $800 \mathrm{~km} \mathrm{~s}^{-1}$ and $400 \mathrm{~km} \mathrm{~s}^{-1}$, respectively (Fig. 5, top). The $600 \mathrm{~km} \mathrm{~s}^{-1}$ and $300 \mathrm{~km} \mathrm{~s}^{-1}$ solar wind components in the activity maximum case (Fig. 5, bottom), were defined in the same way, as a result of a peak wind speed of $u_{\mathrm{r}} \sim 614 \mathrm{~km} \mathrm{~s}^{-1}$ in the 3D domain. 
Table 2. Mass loss rates, $\dot{M}$ and angular momentum loss rates, $\dot{J}$, calculated from the steady-state solutions.

\begin{tabular}{c|cc|cc|c|cc}
\hline \hline Parameter & \multicolumn{2}{|c|}{ HD 1237 } & \multicolumn{2}{c|}{ HD 22049 } & \multicolumn{2}{c}{ HD 147513 } & \multicolumn{2}{c}{ Sun } \\
& ZDI & SH-ZDI & ZDI & SH-ZDI & SH-ZDI & CR 1922(Min) & CR 1962 (Max) \\
\hline$\dot{M}\left[\times 10^{-14} M_{\odot} \mathrm{yr}^{-1}\right]$ & 4.70 & 13.9 & 2.77 & 10.2 & 11.4 & 2.76 & 4.80 \\
$\dot{J}\left[\times 10^{30} \mathrm{erg}\right]$ & 6.77 & 58.0 & 1.10 & 12.3 & 3.66 & 0.40 & 1.10 \\
$\left\langle u_{\mathrm{r}}\right\rangle_{\mathrm{AS}}\left[\mathrm{km} \mathrm{s}^{-1}\right]$ & 679 & 654 & 562 & 493 & 363 & 364 & 171 \\
$\langle n\rangle_{\mathrm{AS}}\left[\times 10^{4} \mathrm{~cm}^{-3}\right]$ & 2.85 & 3.09 & 2.65 & 3.64 & 15.1 & 4.97 & 30.0 \\
$\langle T\rangle_{\mathrm{AS}}\left[\times 10^{6} \mathrm{~K}\right]$ & 2.33 & 1.52 & 1.81 & 1.34 & 1.54 & 1.26 & 0.84 \\
$\langle B\rangle_{\mathrm{AS}}\left[\times 10^{-2} \mathrm{G}\right]$ & 1.94 & 2.08 & 1.82 & 2.23 & 2.98 & 1.64 & 2.63 \\
$\left\langle R_{\mathrm{AS}}\right\rangle\left[R_{*}\right]$ & 12.0 & 19.6 & 10.2 & 15.6 & 7.1 & 6.4 & 6.8 \\
\hline
\end{tabular}

Notes. The additional stellar wind properties represent averages over the resulting Alfvén surface (AS), while $\left\langle R_{\mathrm{AS}}\right\rangle$ corresponds to the mean AS radius in each case.
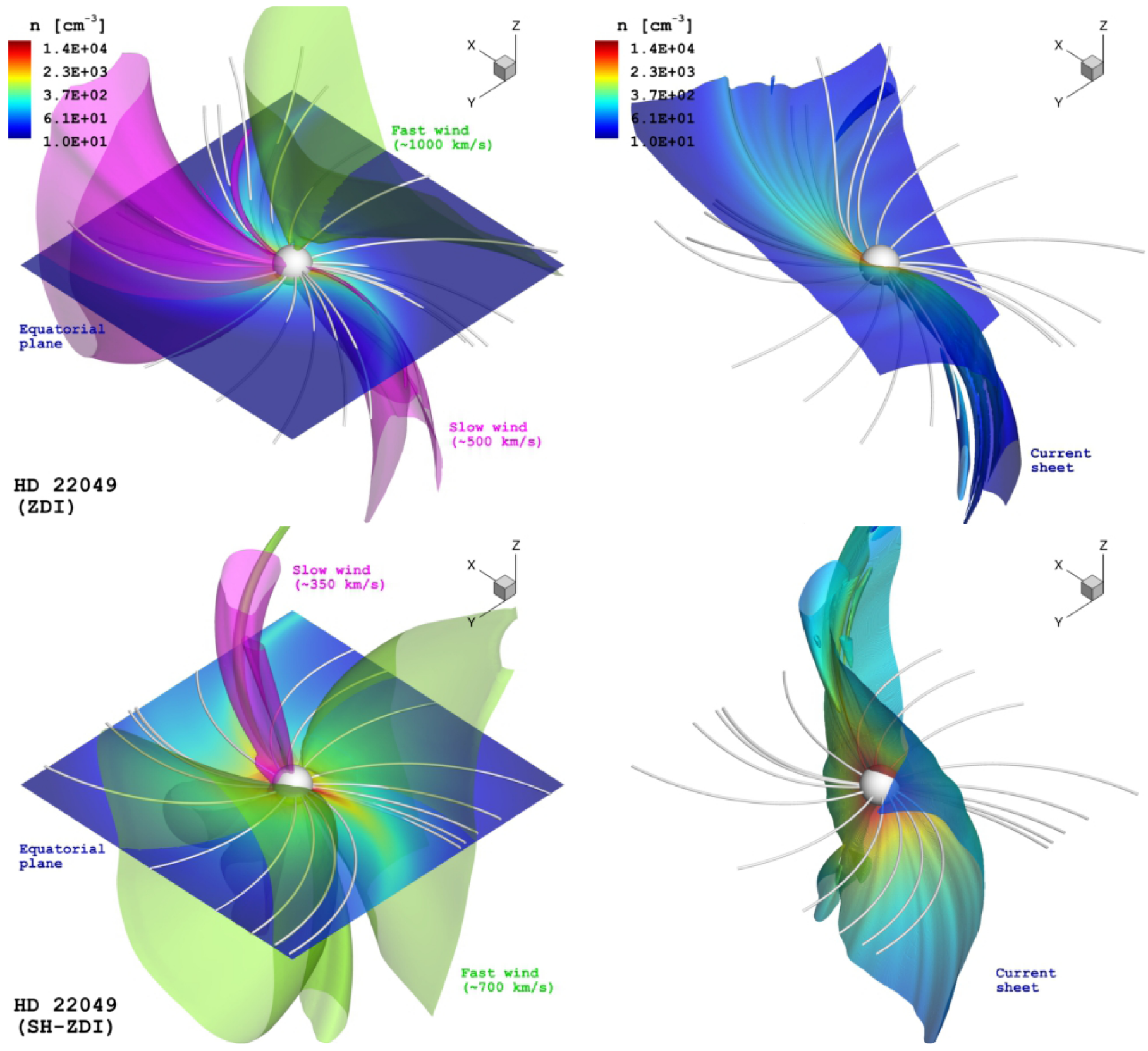

Fig. 6. Simulation results in the IH domain for HD 22049 driven by the ZDI (top) and SH-ZDI (bottom) magnetic field maps. The central white sphere denotes the boundary with the SC domain at $25 R_{*}$ (Sect. 3.1). The density structure of the steady-state solution is displayed on the equatorial plane (left) and the astrospheric current sheet (right). In the left panel, the topology and associated magnitudes of the dominant radial velocity components $\left(u_{\mathrm{r}}\right)$ of the stellar wind are also included (fast: green - slow: magenta). The perspective and density colour scale are preserved among the different panels. Selected 3D magnetic field lines are shown in white. 

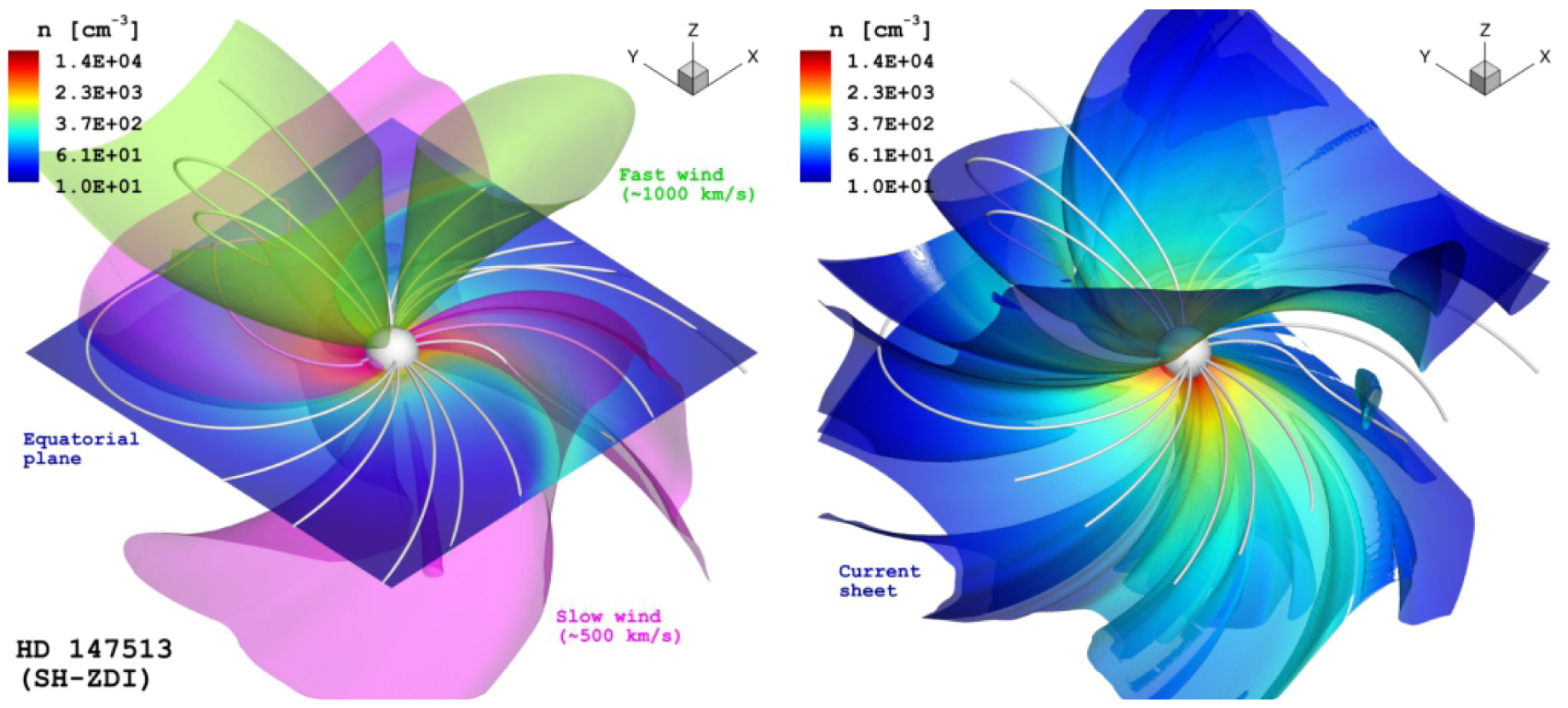

Fig. 7. Simulation results in the IH domain for HD 147513 driven by the SH-ZDI magnetic field maps. The central white sphere denotes the boundary with the SC domain at $25 R_{*}$ (Sect. 3.1). The density structure of the steady-state solution is displayed on the equatorial plane (left) and the astrospheric current sheet (right). In the left panel, the topology and associated magnitudes of the dominant radial velocity components $\left(u_{\mathrm{r}}\right)$ of the stellar wind are also included (fast: green - slow: magenta). Selected 3D magnetic field lines are shown in white.

As can be seen from Fig. 5, the expected global properties and topology of the solar wind (for both activity states), are properly recovered in the simulation. The activity minimum case shows the classical solar wind configuration, with the fast wind emerging from the poles, and the slow wind close to the equator, describing a "ballerina skirt" shape along the heliospheric current sheet (Fig. 5, top right). In the activity maximum case, a more complex solution is obtained, with a 3D structure dominated by the slow component which is no longer restricted to lower latitudes. As the overall velocity of the wind is reduced (compared to the solar minimum case), the fast wind component is nearly inexistent in this solution (Fig. 5, bottom left). Additionally, a dramatic change in complexity can be observed in the heliospheric current sheet (Fig. 5, bottom right), as was expected from the resulting topology of this structure inside the inner domain of the simulation (Sect. 3.1). The density structure of the solar wind is clearly enhanced during the activity maximum solution, as can be compared from the equatorial and current sheet projections in Fig. 5. This is quantified in more detail in Sect. 4.

Figure 6 contains the results for HD 22049, driven by the ZDI (top) and the SH-ZDI (bottom) magnetic field maps. As expected from the SC region (Sect. 3.1), differences in the geometry and the wind properties are developed in this domain ${ }^{5}$. Only one broad fast wind region emerges in the ZDI-driven case, with an associated speed of $u_{\mathrm{r}} \sim 1000 \mathrm{~km} \mathrm{~s}^{-1}$ (Fig. 6, top-left). This wind component is roughly perpendicular to the astrospheric current sheet which, close to the star, displays a tilt of $\sim 45^{\circ}{ }^{6}$, and a rotational drag by the wind at farther distances (Fig. 6, topright). In turn, four such fast wind regions are formed in the SHZDI simulation, displaying a $\sim 30 \%$ reduction of the wind speed (Fig. 6, bottom-left). From this wind regime, the two broader structures are again nearly perpendicular to each side of the astrospheric current sheet, which in this case is almost orthogonal

\footnotetext{
5 See also the 3D animations provided as supplementary material.

6 With respect to the stellar rotation axis (i.e., $z$-axis).
}

to the equatorial plane (Fig. 6, bottom-right). The remaining two fast wind regions appear as collimated jet-like structures, closely aligned with the local orientation of astrospheric current sheet. The latter, as with the solar minimum case (Fig. 1, top), shows a connection with the slow wind region in both simulations of HD 22029, where the denser material is carried away from the star.

The results for HD 147513 inside the IH simulation domain are presented in Fig. 7, where the maximum radial wind speed was $u_{\mathrm{r}} \sim 1040 \mathrm{~km} \mathrm{~s}^{-1}$. Two cone-shaped regions, associated with the fast wind component, appear close the north pole of the star. No southern counterpart for these regions was obtained in the simulation. The topologies of the slow wind component and the astrospheric current sheet, clearly resemble the solar maximum solution in this domain (see Fig. 5, bottom). This is consistent with the results obtained inside the SC module (Sect. 3.1) and with the simulated global properties of the corona in both cases (Alvarado-Gómez et al. 2016). However, as discussed in the first paper of this study, these results could be affected by the relatively low spatial resolution of the SH-ZDI map of HD 147513 (see Hussain et al. 2016). Still, this solution indicates that the coronal structure and wind characteristics may be extremely complex, even for cases with a relatively simple surface field distribution. In this context, scaling relations involving average stellar/magnetic properties and extrapolations, cannot provide complete descriptions of the coronal and wind conditions of a particular system. This is critical for characterising planet-hosting stars, where those specific environmental properties (e.g. coronal emission, stellar wind structure, mass loss, etc.) will strongly affect the exoplanetary conditions of the system.

\subsection{Environment of the HD 1237 system}

The results of the simulations performed on the HD 1237 system, driven by the ZDI and SH-ZDI maps, are presented in Figs. 8 and 9 respectively. Two fast wind structures oriented in opposite directions, appear close to the equatorial region of the 


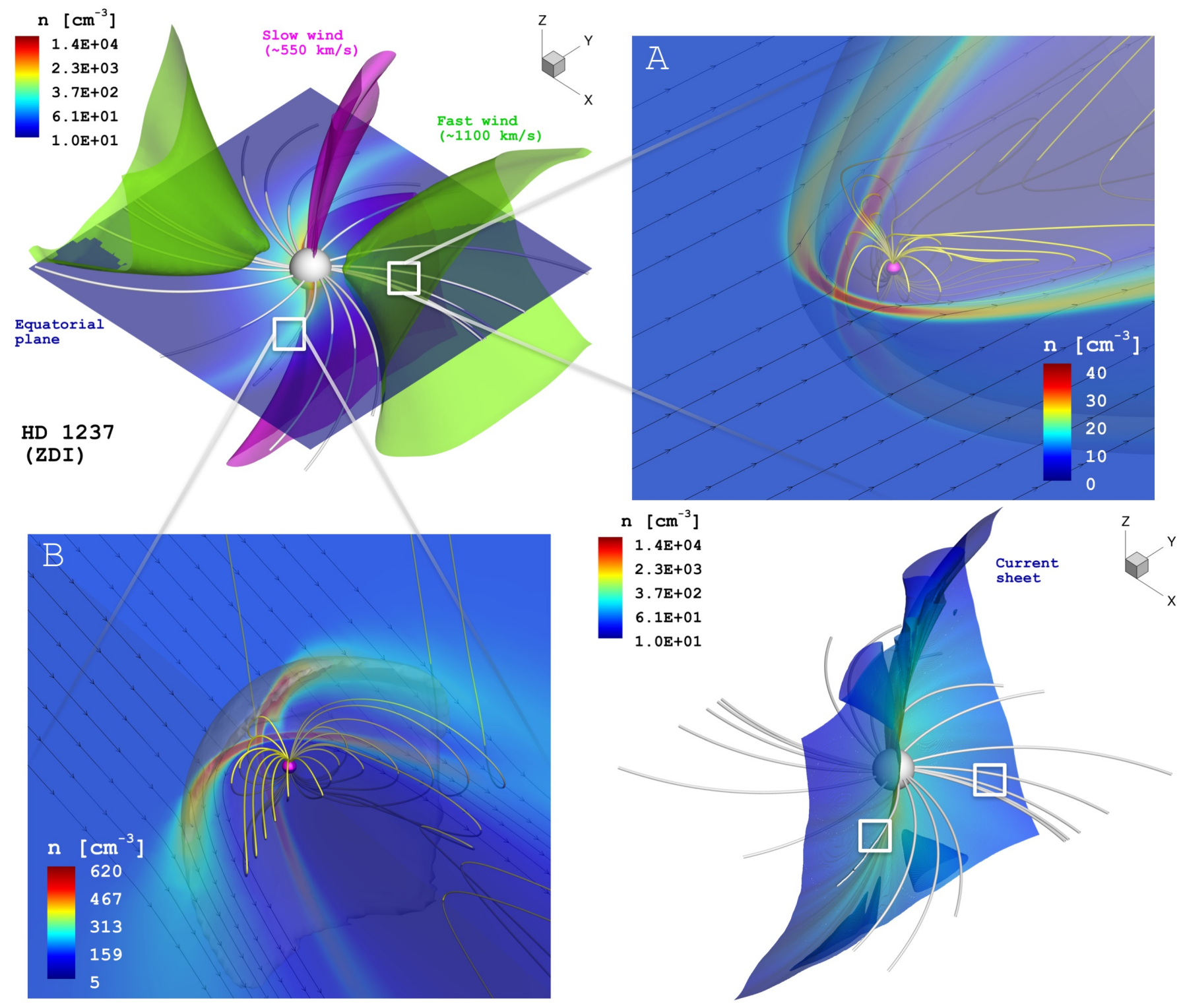

Fig. 8. Simulated environment of the HD 1237 system driven by the ZDI magnetic field map. The structure of the stellar wind and astrospheric current sheet obtained from the IH module, are presented in the top-left and bottom-right panels respectively. The density structure of the steadystate solution is displayed on the equatorial plane (top-left) and the astrospheric current sheet (bottom-right). In the top-left panel, the topology and associated magnitudes of the dominant radial velocity components $\left(u_{\mathrm{r}}\right)$ of the stellar wind are also included (fast: green - slow: magenta). The central white sphere denotes the boundary with the SC domain at $25 R_{*}$ (Sect. 3.1), and selected 3D stellar wind magnetic field lines are shown in white. The two remaining panels contain the simulation results of the GM module, obtained at the locations indicated on the IH domain by the white squares (not to scale). The distance to the star has been taken as the mean orbital separation of this system $(a=0.49$ AU, Naef et al. 2001). The central purple sphere corresponds to the planetary surface $\left(1 R_{\mathrm{p}}\right)$ and selected 3D planetary magnetic field lines are displayed in yellow. The direction of the incident stellar wind is indicated by the black streamlines. The particle density distribution of the solution shows the development of a bow-shock structure in both cases (translucent white shade).

system. These structures are connected to the large, low-latitude coronal holes developed in this system (see Alvarado-Gómez et al. 2016). The wind speed reaches $\sim 1100 \mathrm{~km} \mathrm{~s}^{-1}$ in the ZDIdriven case, dropping to $\sim 900 \mathrm{~km} \mathrm{~s}^{-1}$ in the SH-ZDI simulation. As in some of the previously described cases, the fast wind regions appear roughly perpendicular to the astrospheric current sheet, along which the slow wind region develops. The global topology of the stellar wind is similar between both cases, yet an enhancement in the particle density is again obtained in the SH-ZDI case (see Sects. 3.1 and 3.2). A more quantitative comparison of all cases is presented in the following section.
As described in Sect. 2, the GM module of the SWMF was additionally coupled to the $\mathrm{IH}$ solution to investigate the exoplanetary conditions in relation to the developed stellar wind properties in this system. Two different locations in the IH domain, represented by the white squares in Figs. 8 and 9, were used for this purpose. These locations correspond to a fast wind, low density region (sector A, Figs. 8 and 9, top-right), and a high-density streamer of the stellar wind, close to the astrospheric current sheet (sector B, Figs. 8 and 9, bottomleft). The distance to the central star was $0.49 \mathrm{AU}$ in both cases, matching the mean orbital separation of this system 


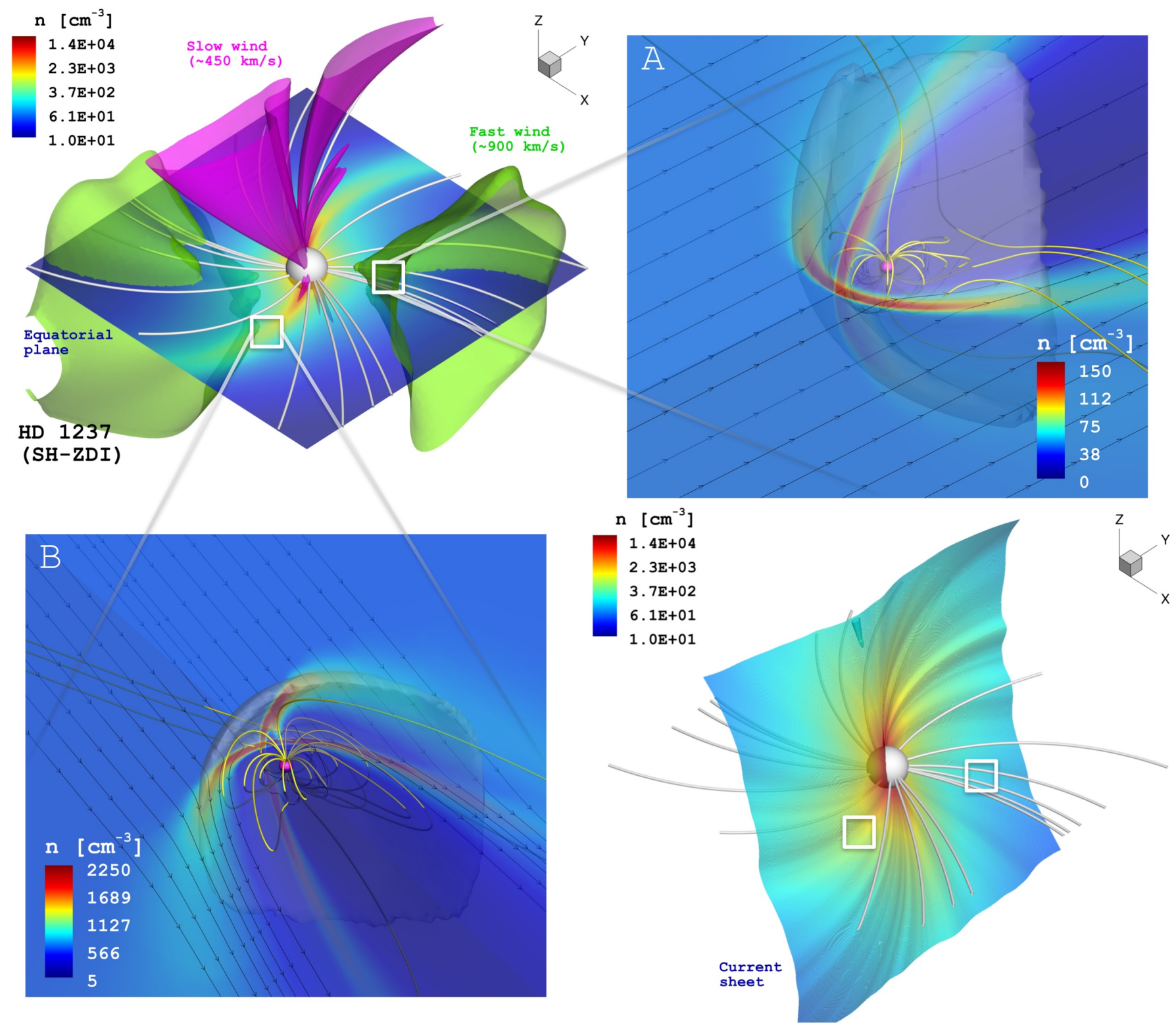

Fig. 9. Simulated environment of the HD 1237 system driven by the SH-ZDI magnetic field map. The structure of the stellar wind and astrospheric current sheet obtained from the IH module, are presented in the top-left and bottom-right panels respectively. The density structure of the steadystate solution is displayed on the equatorial plane (top-left) and the astrospheric current sheet (bottom-right). In the top-left panel, the topology and associated magnitudes of the dominant radial velocity components $\left(u_{\mathrm{r}}\right)$ of the stellar wind are also included (fast: green - slow: magenta). The central white sphere denotes the boundary with the SC domain at $25 R_{*}$ (Sect. 3.1), and selected 3D stellar wind magnetic field lines are shown in white. The two remaining panels contain the simulation results of the GM module, obtained at the locations indicated on the IH domain by the white squares (not to scale). The distance to the star has been taken as the mean orbital separation of this system $(a=0.49$ AU, Naef et al. 2001). The central purple sphere corresponds to the planetary surface $\left(1 R_{\mathrm{p}}\right)$ and selected 3D planetary magnetic field lines are displayed in yellow. The direction of the incident stellar wind is indicated by the black streamlines. The particle density distribution of the solution shows the development of a bow-shock structure in both cases (translucent white shade).

(Naef et al. 2001). The interaction between the magnetised stellar wind and the planetary magnetosphere, led to the development of a bow-shock structure, which self-consistently reacts to the local conditions. A summary of the driving stellar wind properties, and the resulting magnetospheric conditions in both locations, is presented in Table 3 .

A larger response from the magnetosphere was obtained in the SH-ZDI case as expected, given the incident stellar wind properties in the IH domain (see Table 3). In the fast wind region (location A in Figs. 8 and 9), the maximum total pressure at the bow-shock $\left(P_{\mathrm{T}}^{\max }\right)$ appeared $\sim 2$ times larger in the SH-ZDI case compared to the ZDI simulation. This led to $\sim 15 \%$ reduction in the magnetosphere size (in terms of the magnetopause standoff distance $R_{\mathrm{M}}$ ) in the former case compared to the latter. A similar situation was obtained in the simulations at the high-density streamer sector (location B in Figs. 8 and 9). The SH-ZDI case yielded a $\sim 3.7$ times larger $P_{\mathrm{T}}^{\max }$ value, and a $\sim 25 \%$ reduction in $R_{\mathrm{M}}$, in comparison with the ZDI-driven model. On the other hand, the resulting conditions were more extreme in the dense streamer sector (location B), than in the fast wind region (location A), regardless of the driving field distribution. This is evidenced by the $\sim 10$ times increase in the average particle 
Table 3. Parameters of the incident stellar wind and resulting properties inside the GM module for each location.

\begin{tabular}{l|cccc|ccc}
\hline \hline $\begin{array}{l}\text { Location/ } \\
\text { case }\end{array}$ & \multicolumn{4}{|c|}{ Incident stellar wind (IH module) } & \multicolumn{3}{c}{ Global magnetosphere (GM module) } \\
& $n\left[\mathrm{~cm}^{-3}\right]$ & $T\left[\times 10^{5} \mathrm{~K}\right]$ & $\boldsymbol{u}_{\mathrm{sw}}\left[\mathrm{km} \mathrm{s}^{-1}\right]$ & $\boldsymbol{B}[\mathrm{nT}]$ & $R_{\mathrm{M}}\left[R_{\mathrm{p}}\right]^{a}$ & $P_{\mathrm{T}}^{\max }[\mathrm{nPa}]^{b}$ & $\langle n\rangle\left[\mathrm{cm}^{-3}\right]^{c}$ \\
\hline A/ZDI & 10.9 & 5.37 & $(-829.5,804.8,-5.4)$ & $(-16.5,10.5,0.01)$ & 7.7 & 51.5 & 1.13 \\
A/SH-ZDI & 45.5 & 3.40 & $(-641.3,638.2,-0.2)$ & $(-43.7,-28.7,0.31)$ & 6.5 & 101.7 & 3.05 \\
B/ZDI & 160.0 & 1.03 & $(-383.7,466.4,-6.3)$ & $(1.22,-3.31,-0.18)$ & 6.4 & 165.2 & 9.13 \\
B/SH-ZDI & 596.7 & 1.12 & $(-222.5,553.7,-5.8)$ & $(1.15,-23.1,0.60)$ & 4.7 & 618.7 & 35.1 \\
\hline
\end{tabular}

Notes. ${ }^{(a)}$ This value corresponds to the magnetopause standoff distance (i.e. magnetopause day-side separation). ${ }^{(b)}$ Maximum value of the total pressure $\left(P_{\mathrm{T}}=P_{\mathrm{gas}}+P_{\mathrm{dyn}}+P_{\mathrm{mag}}\right)$ at the bow-shock location. ${ }^{(c)}$ Average particle density at a spherical surface with $R=2 R_{\mathrm{p}}$. ${ }^{(\dagger)}$ Cartesian components of the vector quantities are provided.

density $\langle n\rangle$, between locations A and B (see Table 3), calculated at $1 R_{\mathrm{p}}$ above the planetary surface (i.e. over a spherical surface with $R=2 R_{\mathrm{p}}$ ). As can be seen for Figs. 8 and 9, the developed wind structure shows a larger contrast in density than in velocity. This general property was common in the remaining systems considered here and in the solar cases (see Sect. 3.2).

In this context, the results obtained for locations A and B indicate that the process of particle injection into the planetary atmosphere, would be more sensitive to the density structure rather than to the velocity profile of the stellar wind.

Finally, and linked to this last result, we can consider the simulated wind structure and the exoplanetary orbit to investigate the possible magnetospheric radio emission from the exoplanet of this system. The theoretical considerations for this kind of emission have been presented in various papers (e.g. Farrell et al. 1999; Zarka et al. 2001; Grießmeier et al. 2007; Jardine \& Collier Cameron 2008; Nichols 2011; Nichols \& Milan 2016), and extensive observational searches have been performed over the last decade (e.g. Lazio \& Farrell 2007; Lazio et al. 2010a, 2010b; George \& Stevens 2007; Lecavelier et al. 2009, 2011, 2013; Hallinan et al. 2013; Sirothia et al. 2014). For the particular case of HD 1237, Stevens (2005) identified this system as a good candidate for detection, with a mean radio flux of $8 \mathrm{mJy}$ at a peak frequency of $\sim 40 \mathrm{MHz}$, reaching up to $\sim 20 \mathrm{mJy}$ during periastron passage. Lacking better information, Stevens (2005) used reasonable approximations regarding the stellar wind and planetary properties, such as a scaling relation from Wood et al. (2002) between the X-ray flux and the mass loss rate of the host star (see Sect. 4.1), spherical symmetry for the stellar wind, scaling of the planetary magnetic moment and radius, among others. While no better constraints are available for the planetary properties, our data-driven simulations provide a more realistic description of the wind of this star. For this reason, we retained the assumptions made by Stevens (2005) regarding the exoplanet properties of radius and magnetic moment $\mathcal{M}_{\mathrm{p}}$ (i.e., $\left.R_{\mathrm{p}}=R_{\mathrm{J}} \simeq 7.2 \times 10^{9} \mathrm{~cm} ; \mathcal{M}_{\mathrm{p}}=\mathcal{M}_{\mathrm{J}} \simeq 1.6 \times 10^{30} \mathrm{G} \mathrm{cm}^{3}\right)$. This implies that the $40 \mathrm{MHz}$ peak frequency of the expected magnetospheric radio emission remains unaltered (see Stevens 2005). For this analysis we only consider the results from the SH-ZDI simulation, as it provides favourable conditions in terms of increased stellar wind density (see Figs. 8 and 9).

Following Zarka et al. (2001), the emitted radio power from the exoplanet, $R^{\text {pow }}$, will be proportional to the kinetic power $K_{\mathrm{sw}}^{\mathrm{pow}}$ associated with the wind-magnetosphere interaction ${ }^{7}$ (i.e., $R^{\text {pow }}=\alpha K_{\mathrm{sw}}^{\text {pow }}$, with $\left.\alpha=7 \times 10^{-6}\right)$. By combining the simulated stellar wind structure with the assumed exoplanetary properties, we can compute $K_{\mathrm{sw}}^{\mathrm{pow}}$, using the relation

$K_{\mathrm{sw}}^{\mathrm{pow}}=n u_{\mathrm{sw}}^{3} \pi R_{\mathrm{M}}^{2}$,

7 This relation is known as the Radiometric Bode Law. See Zarka et al. (2001) and Lazio et al. (2004).

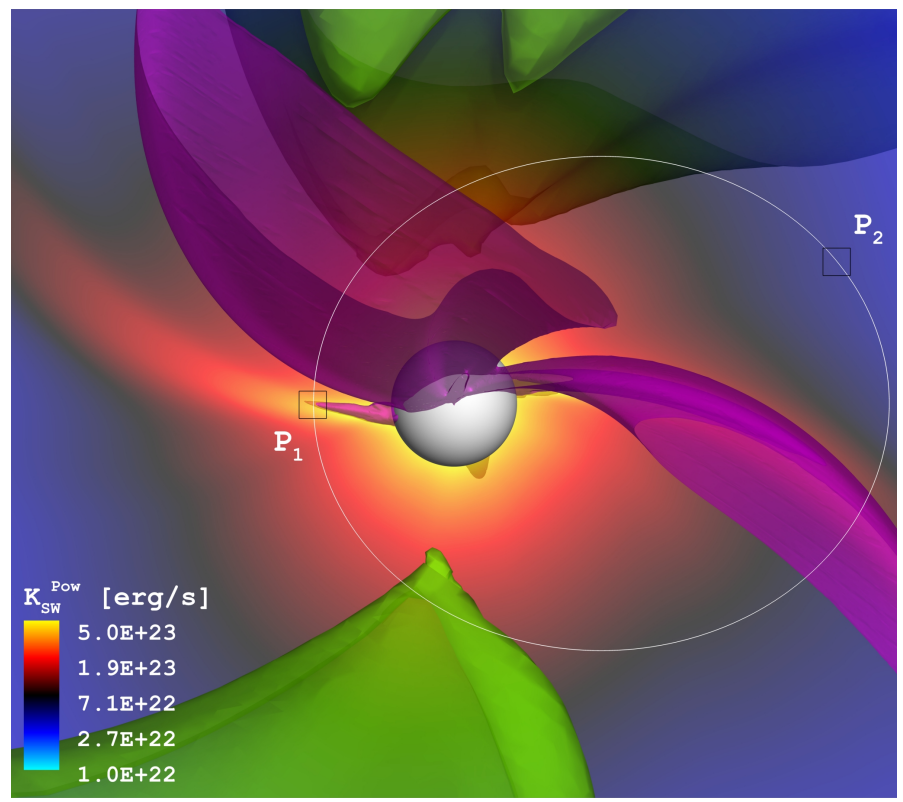

Fig. 10. Pole-on view on the equatorial distribution of the kinetic power $K_{\mathrm{sw}}^{\text {pow }}$ from wind-magnetospheric interaction (see text for details). The green (fast) and magenta (slow) velocity components of the stellar wind are identical as in Fig. 9 (top-left). The configuration of the exoplanet orbit (white ellipse), with respect to the structure of the stellar wind, maximises the radio power at $\mathrm{P}_{1}$ (periastron), and yields a minimum in location $\mathrm{P}_{2}$.

where $R_{\mathrm{M}}$ denotes radius of the magnetosphere, which depends on the local conditions of the wind and the planetary magnetic field (e.g. Table 3). As discussed by Stevens (2005) and references therein, $R_{\mathrm{M}}$ can be expressed as

$R_{\mathrm{M}} \propto\left(\frac{\mathcal{M}_{\mathrm{p}}^{2}}{16 \pi n u_{\mathrm{sw}}^{2}}\right)^{1 / 6}$

and therefore, can be calculated at each point of the simulation domain. However, as the exoplanet location is not arbitrary, only values of $K_{\mathrm{sw}}^{\mathrm{pow}}$ along the planetary orbit will be relevant for the predicted magnetospheric radio emission.

Figure 10 shows the equatorial distribution of $K_{\mathrm{sw}}^{\mathrm{pow}}$, alongside the dominant radial wind components of the SH-ZDI simulation of this system (pole-on view of Fig. 9, top-left). The exoplanet orbit, indicated by the white ellipse, has been constructed using the parameters listed by Naef et al. (2001). The windorbit layout presented in Fig. 10 corresponds to the optimal conditions for magnetospheric radio emission in this system (i.e., periastron passage through a dense wind streamer, location $\mathrm{P}_{1}$ in Fig. 10). In this way, we obtain a maximum emitted radio power 
of $R^{\text {pow }}=\alpha K_{\mathrm{sw}}^{\mathrm{pow}} \simeq 3.5 \times 10^{18} \mathrm{erg} \mathrm{s}^{-1}$, which yields a corresponding radio flux on Earth $^{8}$ of $F_{\mathrm{E}}^{R} \simeq 12 \mathrm{mJy}$. This value is reduced up to a factor of $\sim 10$ in the location marked as $\mathrm{P}_{2}$ in Fig. 10, which does not coincide with apastron in this configuration.

Our maximum radio flux $F_{\mathrm{E}}^{R}$ is roughly half of the previous estimate of $21.5 \mathrm{mJy}$ of Stevens (2005). In turn, the predicted orbital variation in our simulation is more than twice as large as the $\sim 4.5$ factor obtained in this previous work. Given that this previous analysis was performed assuming a stellar wind velocity of $400 \mathrm{~km} \mathrm{~s}^{-1}$ (slower than the value predicted in our simulations at periastron by $\sim 25 \%$ ), the higher radio power obtained by Stevens (2005) must be connected with the assumed stellar wind density (obtained via a spherically symmetric wind with $\dot{M}=85.7 \dot{M}_{\odot}$ ). As will be discussed in the following section, this mass loss rate value is probably overestimated, supporting a lower value of the planetary radio flux. The difference in the orbital variation of $F_{\mathrm{E}}^{R}$ appears as a consequence of a more realistic description of the $3 \mathrm{D}$ stellar wind structure provided by our datadriven simulation.

Finally, the obtained values for the magnetospheric radio emission should be within the expected capabilities of the Square Kilometre Array (SKA), particularly in the lowfrequency band (50 MHz up to $350 \mathrm{MHz}$, see Zarka et al. 2015). The on-sky location of this system $\left[\alpha(\mathrm{J} 2000): 00^{\mathrm{h}} 16^{\mathrm{m}} 16.68^{\mathrm{s}}\right.$, $\delta$ (J2000): $-79^{\circ} 51^{\prime} 04.25^{\prime \prime}$ ], prevents observations with current instrumentation with low-frequency capabilities, such as the Low-Frequency Array (LOFAR) and the Ukrainian T-shaped Radio telescope (UTR-2).

\section{Analysis and discussion}

In a similar manner to Alvarado-Gómez et al. (2016), we use the simulation results to analyse several aspects of the environment of these systems. In this way, we consider the connection between the surface magnetic field properties, and the predicted mass and angular momentum loss rates associated with the wind (Sect. 4.1). In addition, a characterisation of the stellar wind properties at the inner edges of the habitable zones of these systems is presented in Sect. 4.2.

\subsection{Magnetism and mass/angular momentum loss rates}

As with the first paper of this study, this analysis considers the results of our simulations independently (i.e. solar $\min / \max$ and stellar ZDI/SH-ZDI cases). Figure 11 shows the dependence of the simulated mass and angular momentum loss rates $(\dot{M}$ and $\dot{J}$, respectively), with respect to the unsigned radial magnetic flux $\Phi_{\mathrm{Br}}$, averaged over the stellar surface. An approximately linear dependence is obtained for $\dot{M}\left(\propto \Phi^{0.89 \pm 0.08}\right)^{9}$, while a quadratic relation (with increased scatter) is obtained for $\dot{J}\left(\propto \Phi^{2.02 \pm 0.41}\right)$. Given the direct dependence of $\dot{J}$ with $\dot{M}$ (see Cohen et al. 2010; Matt et al. 2012, 2015; Cohen \& Drake 2014; Réville et al. 2015a; Garraffo et al. 2015b), we will focus our discussion on the results obtained for the mass loss rate. A diagram similar to Fig. 11, relating the simulated coronal radiation (e.g. EUV, $\mathrm{X}$-rays) with $\left\langle\Phi_{\mathrm{Br}}\right\rangle_{\mathrm{s}}$ was presented in Alvarado-Gómez et al. (2016).

Unlike the high-energy emission, mass loss rate estimates are only available for 10 Sun-like stars (spectral types G-K). This sample covers activity levels expressed in terms of X-ray

\footnotetext{
8 We have used here the distance to HD 1237 of 17.5 pc (Koen et al 2010).

9 For simplicity, in the following relations $\Phi$ represents $\left\langle\Phi_{\mathrm{Br}}\right\rangle_{\mathrm{s}}$.
}

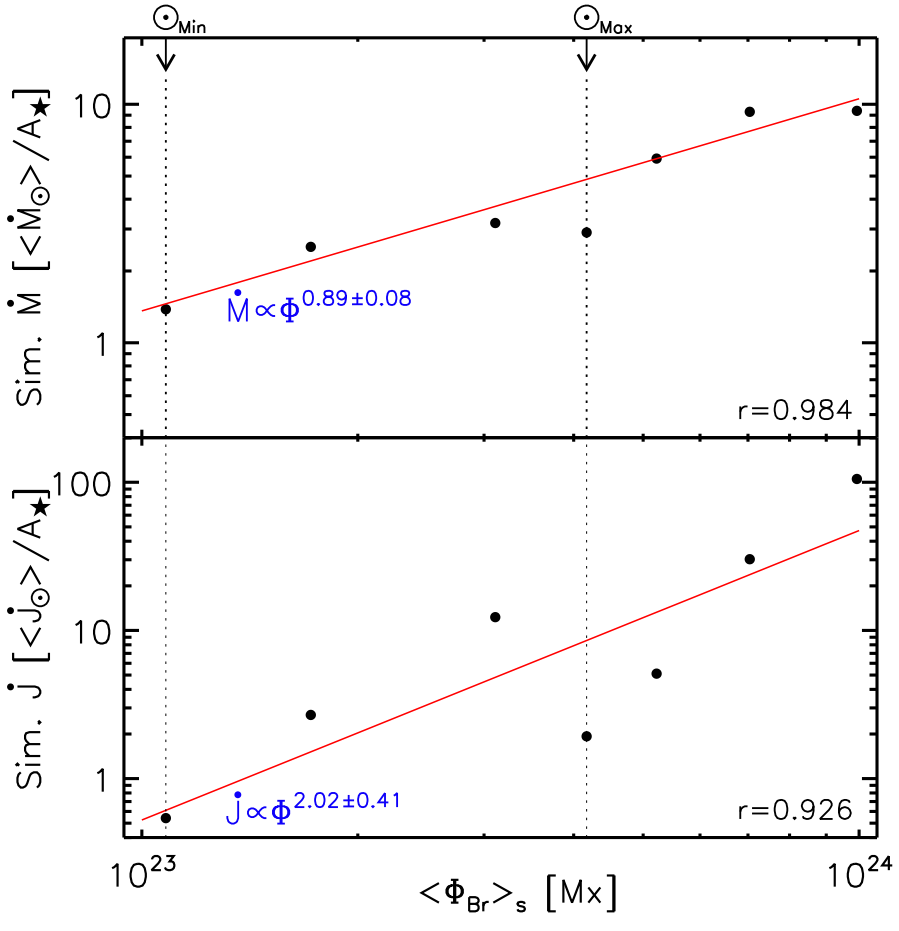

Fig. 11. Simulated mass loss rate $(\dot{M}$, top $)$ and angular momentum loss rate $(\dot{J}$, bottom $)$ as a function of the surface-averaged unsigned radial magnetic flux $\left\langle\Phi_{\mathrm{Br}}\right\rangle_{\mathrm{s}}$. Both quantities are expressed in units of average solar values (Sect. 3.1), normalised by the surface area of each star. Individual points denote the results of each simulation presented in Sect. 3, including the solar cases (indicated by the dashed vertical lines). The red solid lines show power-law fits to the simulated data with the corresponding correlation coefficient, $r$, in each case.

fluxes $\left(F_{\mathrm{X}}\right)$ between $10^{4}$ to few $10^{6} \mathrm{erg} \mathrm{cm}^{-2} \mathrm{~s}^{-1}$, and includes 5 multiple systems ( $\alpha$ Cen, $61 \mathrm{Cyg}, \xi$ Boo, 36 Oph and 70 Oph), and 5 single stars (61 Vir, Sun, $\epsilon$ Ind, $\epsilon$ Eri and $\left.\pi^{1} \mathrm{UMa}\right)$. As mentioned in Sect. 1, these indirect measurements are obtained via the hydrogen wall in the astrosphere of the system, which is detected as extra HI Lyman- $\alpha$ absorption in the UV region of the spectra (Wood et al. 2005a, 2014). In the low and moderate activity regime $\left(F_{\mathrm{X}}<10^{6} \mathrm{erg} \mathrm{cm}^{-2} \mathrm{~s}^{-1}\right)$, a mass loss-activity relation in the form of $\dot{M} \propto F_{\mathrm{X}}^{1.34 \pm 0.18}$ was suggested by Wood et al. (2005a). This relation appears to break for the high-activity end $\left(F_{\mathrm{X}}>10^{6} \mathrm{erg} \mathrm{cm}^{-2} \mathrm{~s}^{-1}\right)$, where $\xi$ Boo A and $\pi^{1}$ UMa have been recently located with mass loss estimates $\dot{M}<\dot{M}_{\odot}$ for both stars (Wood et al. 2014) ${ }^{10}$.

We can relate these observational studies with our simulations, by combining the $\dot{M}-\Phi$ relation shown in Fig. 11, with the $L_{\mathrm{X}} \propto \Phi^{1.06}$ dependence obtained in Alvarado-Gómez et al. (2016). The latter becomes slightly more steep when expressed in terms of $F_{X}$ (i.e. $F_{X} \propto \Phi^{1.13}$ ). By removing the dependence on $\Phi$, we obtain a simulated mass loss-activity relation in the form of $\dot{M}_{\text {(sim) }} \propto F_{\text {X(sim) }}^{\gamma}$ with $\gamma=0.79_{-0.15}^{+0.19}$, considerably flatter than the observed one. However, we stress that this is the first time that this relation is self-consistently constructed in a model, by computing the X-ray coronal emission and mass loss rate associated with the stellar wind, using the same data-driven numerical

10 Two very active M dwarfs, Proxima Cen (upper limit) and EV Lac, are also located in this region, with small (absolute) mass loss rate values $\left(\dot{M} \leq \dot{M}_{\odot}\right)$. Their location in Wood et al. (2014) diagram is due to their very small surface areas $\left(\sim 0.023 A_{\odot}\right.$ and $0.123 A_{\odot}$, respectively). 
simulation. To understand the differences in these relations, we need to consider several aspects connected to our simulations and the observations. A comprehensive summary is listed below:

- As presented in Sect. 3, the results from the solar simulations are consistent with the observational data. This is not only the case for the mass loss rate (Sect. 3.1) but also for the topology and physical properties of the solar wind during activity minimum and maximum (Sect. 3.2, Fig. 5). Similar results were obtained for the simulated coronal structure for both activity states (Alvarado-Gómez et al. 2016).

- For the stellar systems considered here, an observational estimate of the mass loss rate is only available for HD 22040 ( $\epsilon$ Eri, Wood et al. 2002). As can be seen from Table 2, the ZDI-driven simulation predicts an absolute mass loss rate $\dot{M}_{\text {ZDI }} \simeq \dot{M}_{\odot}$, while the SH-ZDI case leads to $\dot{M}_{\mathrm{SH}-\mathrm{ZDI}} \sim$ $5 \dot{M}_{\odot}{ }^{11}$. This last value differs by a factor of 6 from the estimation from astrospheric Lyman- $\alpha$ absorption $\left(\dot{M}_{\mathrm{Ly}-\alpha} \simeq\right.$ $\left.30 \dot{M}_{\odot}\right)$.

For the other two stars, HD 1237 and HD 147513, Stevens (2005) derived relatively high $\dot{M}$ values $\left(\sim 86 \dot{M}_{\odot}\right.$ and $105 \dot{M}_{\odot}$, respectively), by using an earlier version of the mass loss-activity relation proposed in Wood et al. 2002 (i.e. $\left.\dot{M} \propto F_{\mathrm{X}}^{1.15 \pm 0.20}\right)$. As described before, the range of validity of this relation was revisited by Wood et al. (2005a) and Wood et al. (2014), indicating a break for $F_{\mathrm{X}}>10^{6} \mathrm{erg} \mathrm{cm}^{2} \mathrm{~s}^{-1}$, with evidence in support of much smaller $\dot{M}$ values for stars in this activity regime. This evidence is not only given directly by the astrospheric detections of $\xi$ Boo $\mathrm{A}$ and $\pi^{1} \mathrm{UMa}$ (Wood et al. 2014), but also indirectly, by similar UV observations of a considerable number of active stars yielding non-detections (Wood et al. 2005b). Both stars, HD 1237 and HD 147513, have X-ray fluxes above this empirical $F_{\mathrm{X}}$ threshold (by factors of 2.1 and 1.9, respectively), thus the values listed in Stevens (2005) are probably overestimated.

- In the case of HD 22049, this discrepancy may be addressed by enhancing the base conditions in the simulation of this star. While this certainly would increase the mass loss rate (see Cohen \& Drake 2014), this would also imply a different prediction for the coronal emission in all energy bands (e.g. EUV, SXR). As discussed in Alvarado-Gómez et al. (2016), the SH-ZDI simulation of this system provides reasonable agreement in both, EUV and X-rays, to the estimated coronal conditions via spectral synthesis diagnostics (Sanz-Forcada et al. 2011). Still, further adjustments will be explored for this system in a future systematic approach, in order to improve the balance in the coronal heating (i.e. to the Emission Measure distribution EM, see Ness \& Jordan 2008; Alvarado-Gómez et al. 2016), as well as to refine the predictions of the $\dot{M}$ value. It might even be necessary to include reconnection events as an additional (or dominant) heating mechanism, which appear to drive the coronal conditions in very active stars such as HD 22049 (see Drake et al. 2000). As a drawback, this procedure introduces additional degrees of freedom for the model results, which then complicates the consistent comparison with the reference the solar cases (and with additional stellar simulations).

- Another possibility is related to a temporal dependence of the mass loss-activity relation. In the case of the Sun, it is well known that the coronal emission is enhanced by one order of magnitude over the course of the 11-yr activity cycle (Hathaway 2015). In turn the solar mass loss rate shows little correspondence with the activity state, fluctuating within a

11 The reasons for this relative difference are discussed in Sect. 3.1. factor of $\sim 2$ around a mean value of $\dot{M}_{\odot} \simeq 2 \times 10^{-14} M_{\odot} \mathrm{yr}^{-1}$ (Sect. 3.1, see also Cohen 2011). While there is at least one single star showing a similar cycle-induced ${ }^{12}$ pattern as the Sun in X-rays ( Horologii, see Sanz-Forcada et al. 2013), there is no evidence suggesting that stellar mass loss rates must be cycle-independent (or as near-to-independent as the Sun is). Therefore, variations of 1-2 orders of magnitude in $\dot{M}$, over the course of any possible activity cycle (or due to cycle-dominated transients such as coronal mass ejections CMEs, see Drake et al. 2013), cannot be excluded from the mass loss-activity relation. This would provide a natural explanation for the observed break in this relation, at the regime of high coronal activity.

- Given the magnetic nature of these two processes in Sunlike stars (coronal activity and mass loss), any time dependence of the mass loss-activity relation should be connected with the temporal evolution of the stellar magnetic field. Unfortunately, for the two stars considered in this work located above the break in this relation (HD 1237 and HD 147513), only single-epoch ${ }^{13}$ surface magnetic field reconstructions using ZDI are available (Alvarado-Gómez et al. 2015; Hussain et al. 2016).

On the other hand, a long-term ZDI monitoring campaign of HD 22049 is currently being carried out by the BCool ${ }^{14}$ collaboration. Six large-scale magnetic field maps have been recovered over a period of $7 \mathrm{yr}$ (2007-2013, see Jeffers et al. 2014). As described in the first paper of this study, we have used the set of HARPSpol observations available for this star so far (acquired in 2010, Piskunov et al. 2011), to generate the ZDI maps driving the simulations. This was done to ensure a consistent comparison with ZDI-driven models of the other two stars (whose maps were recovered also using HARPSpol data), by applying the same procedures and criteria in the reconstructions (see Alvarado-Gómez et al. $2015,2016)$, minimising at the same time the effects introduced by driving the simulations using maps from different instruments. By relaxing these last requirements, we can make an order-of-magnitude estimate of the possible temporal variations in $\dot{M}$ and $F_{\mathrm{X}}$ of HD 22049, due to the longterm evolution of its large-scale magnetic field. For this we use the ZDI information provided by Jeffers et al. (2014), together with the $F_{\mathrm{X}}-\Phi$ dependancy derived from the results in Alvarado-Gómez et al. (2016), and the $\dot{M}-\Phi$ relation presented in Fig. 11.

Following this procedure, the recovered large-scale field distributions indicate an approximate change in $\left\langle\Phi_{\mathrm{Br}}\right\rangle_{\mathrm{s}}$ by a factor of $\sim 2$ in a time-scale of at least $5 \mathrm{yr}^{15}$. This would imply variations in $F_{\mathrm{X}}$ and $\dot{M}$ up to factors of $\sim 2.4$ and 2.1 , respectively. We stress here that this calculation corresponds

12 The cycle length $(\sim 1.6 \mathrm{yr})$ is much shorter than the solar cycle, as expected from the relatively young age of the star ( $\sim 500-740 \mathrm{Myr}$, see Sanz-Forcada et al. 2013).

13 Actually, two independent ZDI maps were recovered for HD 1237 separated by 5 months. However, the quality of the second map was far from optimal, due to a very limited phase coverage (see Alvarado-Gómez et al. 2015). Still, this partial field reconstruction indicated very similar properties as with the robust ZDI maps of the first epoch, which have been used to drive the models presented here.

${ }^{14}$ http://bcool.ast.obs-mip.fr

15 This is obtained by considering the largest difference (including uncertainties) in either, the maximum $\left(B_{\max }\right)$ or the mean $\left(B_{\text {mean }}\right)$ magnetic field values listed by Jeffers et al. (2014). For the former, this occurred between the epochs of 2007 and 2012, while for the latter this was visible among the maps of 2008 and 2013. 
to a first-order approximation, as we are neglecting several important elements such as the field topology, complexity, missing magnetic flux, and map incompleteness, among others, which are known to influence the predictions of $F_{\mathrm{X}}$ and $\dot{M}$ based on ZDI maps (e.g. Arzoumanian et al. 2011; Garraffo et al. 2013, 2015a,b; Lang et al. 2014; Alvarado-Gómez et al. 2016). Nevertheless, archival X-ray observations of this star, available at the Nearby X-ray and Extreme UV Emitting Stars (NEXXUS 2) database ${ }^{16}$ (Schmitt \& Liefke 2004), indicate a variation in $F_{\mathrm{X}}$ within a time-scale of 10 years compatible with our previous estimate. Such change is sufficient to move HD 22049 above the previously mentioned threshold in the mass-loss activity relation $\left(F_{\mathrm{X}}=10^{6} \mathrm{erg} \mathrm{cm}^{-2} \mathrm{~s}^{-1}\right)$. Assuming a physical origin for this apparent break, the variation in $F_{\mathrm{X}}$ would imply a decrease in the mass loss-rate value of HD 22049, much larger than the one estimated above and reaching similar values as the ones predicted by our simulations.

- Last but not least, we must also consider the uncertainties associated with the observational estimates of mass loss rates. As explained by Wood et al. (2005a) and Wood et al. (2014), robust astrospheric detections require precise knowledge of the physical structure of the local ISM (e.g. the case of $\lambda$ And, see Malamut et al. 2014). This includes column densities, kinematics, and metal depletion rates (Redfield \& Linsky 2004a; Redfield \& Falcon 2008), together with local temperature and turbulent velocities (Redfield \& Linsky 2004b), interpreted within a particular model of the morphology of the ISM (see Redfield \& Linsky 2008, 2015; Gry \& Jenkins 2014). While these studies have provided a detailed characterisation of the local ISM, intrinsic uncertainties and additional observational issues connected with the astrospheric detections, can certainly modify the estimated mass loss rates by large amounts (conservatively, by factors 2-3; see Linsky \& Wood 2014; Wood et al. 2002).

Some of the possibilities discussed before are currently being explored and will be presented in a future paper. This study will follow the same data-driven methodology presented here, applied to $70 \%$ of the stars with astrospheric detections (Wood et al. 2014) and with surface magnetic fields distributions recovered by $\mathrm{ZDI}^{17}$ (see Vidotto et al. 2016 and references therein).

\subsection{Stellar winds and habitable zones}

Finally, we can use our 3D simulation results, to characterise the stellar wind conditions in the estimated boundaries of the habitable zones (HZ) of these systems. For the latter, we take advantage of the information provided in the Habitable Zone Gallery ${ }^{18}$ (HZG, Kane \& Gelino 2012), regarding the optimistic and conservative calculations of the HZ (as defined in Kopparapu et al. 2013, 2014). We restrict our analysis to the inner edges of the $\mathrm{HZs}$, given the enhancement of various stellar wind properties (e.g. density, magnetic field) closer to the star, which could influence the very definition of this boundary. Reference calculations at $1 \mathrm{AU}$ are also performed for all the simulated cases. In the case of the Sun, the inner edges of the HZ are extremely close to

\footnotetext{
16 http://www.hs.uni-hamburg.de/DE/For/Gal/Xgroup/ nexxus/nexxus.html

17 Additional ZDI maps have been recovered using the NARVAL spectro-polarimeter (Aurière 2003), from observations acquired during 2015 (Program ID: L151N08 - PI: Morin).

18 http://www.hzgallery.org/
}

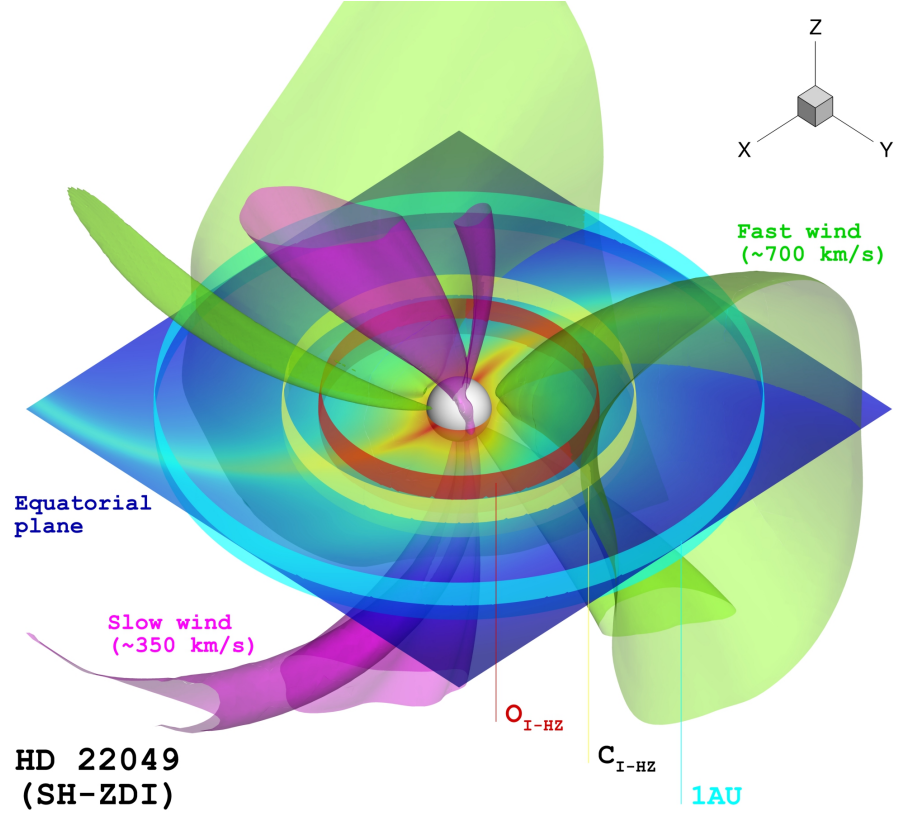

Fig. 12. Stellar wind characterisation at the optimistic $\left(\mathrm{O}_{\mathrm{I}-\mathrm{HZ}}\right.$, red $)$ and conservative $\left(\mathrm{C}_{\mathrm{I}-\mathrm{HZ}}\right.$, yellow) inner edges of the HZ of HD 22049. Similarly, the 1 AU boundary is used as reference (cyan). In all cases, we consider rings extending to $\pm 5^{\circ}$ in inclination from the equatorial plane (slightly exaggerated in the figure for visualisation purposes). The wind structure is the same as in Fig. 6 (bottom-left), rotated by $90^{\circ}$ clockwise.

$1 \mathrm{AU}$, thus we consider instead the semi-major axes of Mercury $\left(a_{\mathrm{M}} \simeq 0.39 \mathrm{AU}\right)$ and Venus $\left(a_{\mathrm{V}} \simeq 0.72 \mathrm{AU}\right)$.

As an example, Fig. 12 shows the optimistic $\left(\mathrm{O}_{\mathrm{I}-\mathrm{HZ}}\right.$, red $)$ and conservative ( $\mathrm{C}_{\mathrm{I}-\mathrm{HZ}}$, yellow) inner edges of the HZ of HD 22049. These are displayed alongside the $1 \mathrm{AU}$ boundary (cyan), and the stellar wind structure developed in the IH domain of the SH-ZDI simulation (Sect. 3.2, Fig. 6). As presented in the visualisation, we consider rings extending $\pm 5^{\circ}$ in inclination from the equatorial plane, to calculate different stellar wind properties and their variation (e.g., min, mean, $\max$ ) at the distance of interest (see Table 4). In this manner the characterisation preserves the threedimensional structure of the stellar wind, within the inclination range where the vast majority of exoplanets have been detected to date ${ }^{19}$ (Han et al. 2014).

The simulated solar wind parameters at $1 \mathrm{AU}$ are consistent with the nominal measurements performed by the Advanced Composition Explorer (ACE) spacecraft during different periods of activity. However, the wind particle density $(n)$ is too high for the solar maximum simulation ${ }^{20}$, which is connected to the overestimated mass loss rate for this epoch (see Sect. 3.1). Still, the simulated parameters are within feasible limits during periods of high activity (which would also involve transient events such as CMEs, see Webb \& Howard 2012; Cohen et al. 2014).

The results listed in Table 4 allow a quick assessment of the circumstellar conditions among the different stellar cases and the Sun. For this, one can use the total pressure values associated to the stellar wind in each case $\left(P_{\mathrm{T}}\right)$. This quantity encompasses the thermal properties of the incident plasma $\left(P_{\text {gas }}=n k_{\mathrm{B}} T\right.$, with $k_{\mathrm{B}}$ as the Boltzmann constant), the dynamic pressure of the

\footnotetext{
19 http://exoplanets.org/

20 This is obtained by comparing the simulated average particle density, $n$, with daily ACE measurements during the CR 1962 (Apr.-May 2000). See http://www.srl.caltech.edu/ACE/ASC/ level2/new/intro.html
} 
wind $\left(P_{\mathrm{dyn}}=n u_{\mathrm{sw}}^{2} / 2\right)$, and the contribution from the magnetic pressure $\left(P_{\text {mag }}=B^{2} / 8 \pi\right)$. For instance, if the Earth were located at $1 \mathrm{AU}$ from HD 1237, the total pressure acting over the magnetosphere would be on average one order of magnitude larger than the nominal conditions around the Sun. In particular sectors of the orbit (i.e., dense streamers in the SH-ZDI simulation), this value can reach more than 2 orders of magnitude of difference compared to the ambient solar wind. This would imply a reduction in the magnetosphere size by a factor ${ }^{21} \sim 2.2$. While variations of this order have been observed in the actual magnetosphere of the Earth (see Pulkkinen 2007), this estimate does not include the effects from magnetic reconnection at the magnetopause (Frey et al. 2003), or due to transient events such as CMEs (Khodachenko et al. 2007; Cohen et al. 2014, 2011), which can disrupt the magnetospheric structure. This is extremely important in the case of highly active stars, as these transient events may even dominate completely the mass loss rate, wind, and energetic properties of the environment in these systems (see Drake et al. 2013).

Finally, this approach can be used to consider the stellar wind properties of the host-star to improve the different estimates of the HZs of these and other systems. One possibility might involve the inclusion into the $\mathrm{HZ}$ of a minimum planetary magnetic moment (consistent with the simulated stellar wind conditions), in order to sustain a magnetosphere up to a certain height (e.g. a few planetary radii). This new characteristic of the HZ boundary could even depend on the specific level of coronal activity of the planet-host (which can also be constructed using ZDI-driven models, see Alvarado-Gómez et al. 2016). This is important due to the fact that in the case of very active planet-hosts (specially M-dwarfs), the magnetic shielding has to compensate the atmospheric expansion induced by the enhanced high-energy emission of the star (e.g. EUV, X-rays, see Lammer et al. 2007), and stronger CMEs with an increased impact rate (see Kay et al. 2016). Such dynamic characterisation of the $\mathrm{HZ}$ is out of the scope of this paper but will be further explored in a future parametric study, including also additional systems for which ZDI maps are available.

\section{Summary and conclusions}

We carried out elaborated simulations of the stellar wind and inner astrospheric structure of three planet-hosting stars (HD 22049, HD 1237, and HD 147513), using the SWMF (Tóth et al. 2005, 2012). This paper complements the study presented in Alvarado-Gómez et al. (2016), which contains the results of the coronal structure modelling of these systems. Steady-state solutions were obtained for two coupled simulation domains, ranging from $1-30 R_{*}$ (SC domain) and from $25-215 R_{*}$ (IH domain). Large-scale magnetic field maps of these stars, recovered with Zeeman-Doppler imaging, serve to drive the solutions inside the SC domain, which are coupled self-consistently for a combined solution in the IH domain. A summary of our results and main conclusions is provided below:

- Following Alvarado-Gómez et al. (2016), simulations driven by two sets of similar large-scale magnetic field distributions (i.e. ZDI and SH-ZDI) were compared. It is worth noting that both sets of magnetic field maps provided equivalently good fits to the observations and showed substantial similarities in the overall structure of the stellar wind. However,

\footnotetext{
${ }^{21}$ This is estimated using Eq. (2), replacing the dynamical pressure of
} the wind $\left(n u_{\mathrm{sw}}^{2}\right)$ for the total pressure $P_{\mathrm{T}}$. several differences in the magneto-hydrodynamic properties of the solutions were found, including $\sim 10-30 \%$ denser and $\sim 25-35 \%$ colder stellar winds in the SH-ZDI solutions compared to the ZDI cases. In addition, the SH-ZDI simulations led to larger values in the average Alfvén surface size (by a factor of $\sim 1.5$ ), the mass loss rate $\dot{M}$ (by a factor of $\sim 3-4$ ), and the angular momentum loss rate $\dot{J}$ (by roughly one order of magnitude). Therefore, the values listed in Table 2 should be actually interpreted as predicted ranges from this ZDIdriven model.

These variations arise as a consequence of the available magnetic energy to heat the corona and accelerate the wind, which in turn, relates to the different field strengths and map completeness provided by the ZDI and SH-ZDI reconstructions. This strongly differs from previous studies where older implementations of the numerical code used here are considered, and where the completeness in the driving magnetic field distributions yield no significant changes in the wind structure (e.g. Vidotto et al. 2012; see Sect. 3.1).

- The results from two different solar simulations, covering activity minimum (CR 1922) and maximum (CR 1962), were also considered. We showed that this numerical framework properly recovers the expected structure of the solar wind, including thermodynamical properties (e.g. density, temperature), mass loss and angular momentum loss rates $\left(\dot{M}_{\odot}\right.$ and $\dot{J}_{\odot}$, respectively; see Table 2 ), and global topology during each activity state (Figs. 1 and 5). However, the solar maximum simulation showed an over-enhanced plasma density at $1 \mathrm{AU}$ (Sect. 4.2, Table 4), as a consequence of an overestimated mass loss rate (by $\sim 40 \%)^{22}$. This is interpreted as the result of a considerable fraction of missing mixed polarity regions in the driving magnetogram, which was artificially degraded for a more consistent comparison with the stellar cases (see Alvarado-Gómez et al. 2016).

- In general, the stellar wind solutions showed a clear relation with the driving magnetic field distribution, and the developed coronal structure in each case. For HD 22049 (Figs. 2 and 6) and HD 1237 (Figs. 3, 8 and 9) various fast-wind regions appeared self-consistently in the simulations, nearly perpendicular to the astrospheric current sheet structure (defined by $B_{\mathrm{r}}=0$ ), and with a spatial correspondence with the dominant features in their lower corona (e.g. coronal holes, see Alvarado-Gómez et al. 2016). The radial wind velocity in these regions reached up to $\sim 1100 \mathrm{~km} \mathrm{~s}^{-1}$ in the ZDI simulations, dropping to $\sim 700 \mathrm{~km} \mathrm{~s}^{-1}$ in the $\mathrm{SH}-\mathrm{ZDI}$ cases (see Sect. 3.2).

- On the other hand, the simulation of HD 147513 yielded a much more complex wind solution (Figs. 4 and 7), compared to what could have been expected from the simple magnetic field distribution driving the simulation (Alvarado-Gómez et al. 2016). A highly warped astrospheric current sheet was obtained in this case, over which a dominant slow-wind component $\left(u_{\mathrm{r}} \simeq 500 \mathrm{~km} \mathrm{~s}^{-1}\right)$ was developed. While these results could be affected by the comparatively low-resolution of the SH-ZDI map driving the simulation (see Hussain et al. 2016), this example indicates that numerical descriptions based on first order extrapolations of surface magnetic field properties alone, cannot provide a complete picture of the wind complexity in a given system (e.g. Matt et al. 2012).

${ }^{22}$ This corresponds to a very rough estimate, as it relies on the single spatial point measurements and location of Voyager II as reference (http://voyager.jpl.nasa.gov/mission/weekly-reports/ index.htm). 
J. D. Alvarado-Gómez et al.: Stellar winds and inner astrospheres

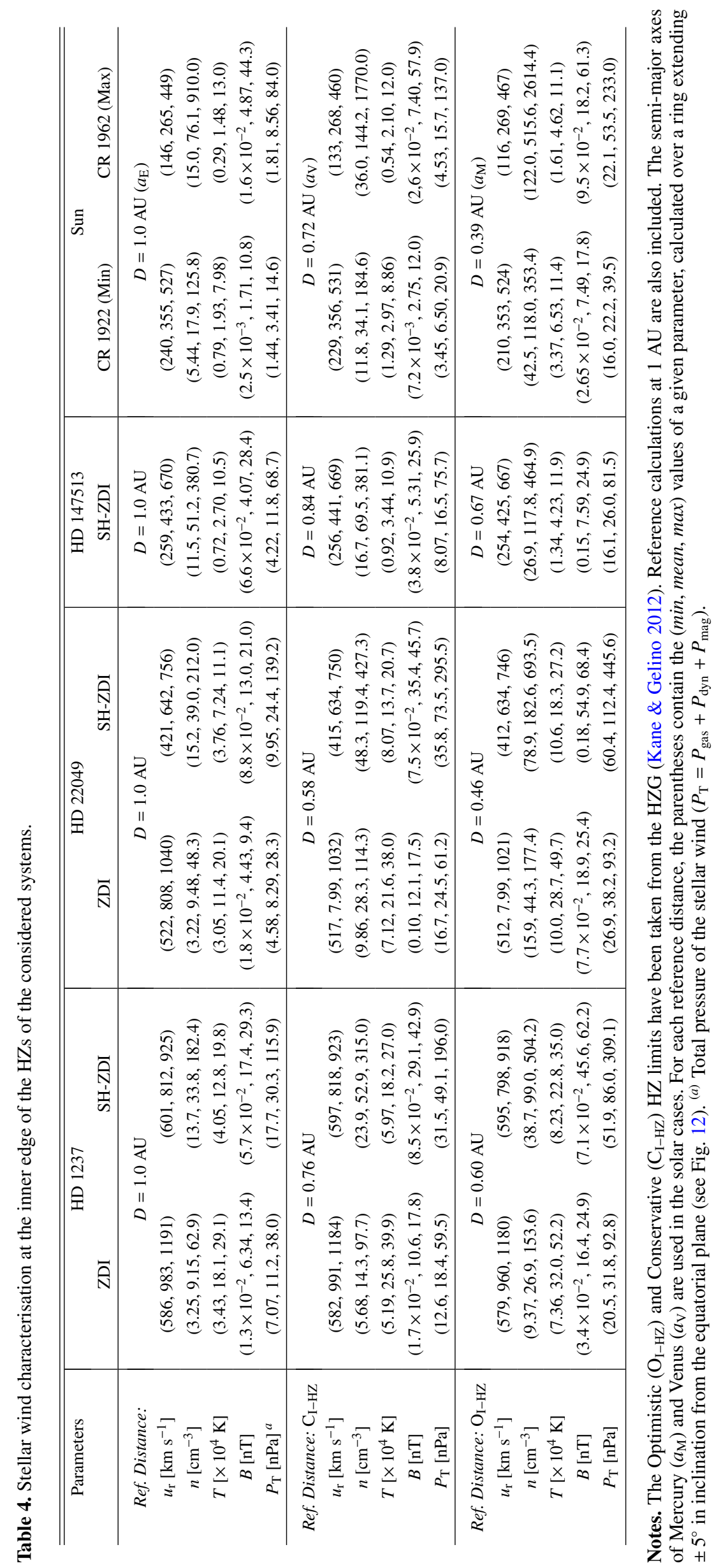


- For HD 1237 we investigated in detail the wind environment and the conditions experienced by the exoplanet of this system (Sect. 3.3). For this purpose we additionally coupled the GM module of the SWMF, to the developed wind structure inside the IH domain. For each simulation (i.e. ZDI and SHZDI cases), two representative spatial locations were considered (Figs. 8 and 9). These included a low-density, fast wind stream, and a high-density, slow wind region. This analysis showed that the density structure of the stellar wind dominates, over the wind velocity, the process of particle injection into the planetary atmosphere (see Table 3 ). This is a consequence of the large density gradients obtained in the wind solutions (i.e., dense streamers with increments up to 4 orders of magnitude in $n$ ), compared to the relatively narrow range of resulting radial wind speeds (with variations only up to a factor of 2 in $u_{\mathrm{r}}$ for the entire 3D domain).

- Following Stevens 2005, we additionally calculated the amount of exoplanetary radio emission from the windmagnetospheric interaction in this system. We obtained a maximum radio flux on Earth of $F_{\mathrm{E}}^{R} \simeq 12 \mathrm{mJy}$ at $40 \mathrm{MHz}$, associated with a high-density streamer crossing during periastron passage (at $0.25 \mathrm{AU}$, Naef et al. 2001). This value is reduced by an order of magnitude during the orbital motion of the planet (approximately at 2/3 of a right-hand oriented orbit with respect to periastron, see Fig. 10). Our maximum emission prediction is lower by a factor of $\sim 2$ compared to the estimates of Stevens (2005), which were based on the mass loss-activity relation of Wood et al. 2002, and the assumption of a spherically symmetric wind. Given the system's low declination, SKA is the only facility which could robustly detect and analyse this emission.

- From our simulations, and applying the methodology explained in Cohen \& Drake (2014) and Garraffo et al. (2015b), we calculated the mass loss rate, $\dot{M}$, and angular momentum loss rate, $\dot{J}$, in these systems. We obtained absolute $\dot{M}$ values, ranging from approximately $1 \dot{M}_{\odot}$ up to $\sim 7 \dot{M}_{\odot}$, and $\dot{J}$ within a broader range of $\sim 1-60$ times the solar prediction (Table 2). In combination with the results for the coronal structure (Alvarado-Gómez et al. 2016), we constructed, for the first time, a fully simulated mass loss-activity relation, expressed as $\dot{M}_{(\mathrm{sim})} \propto F_{\mathrm{X}(\mathrm{sim})}^{\gamma}$ with $\gamma=0.79_{-0.15}^{+0.19}$. A thoughtful discussion is presented in Sect. 4.1, comparing this result with the observational relation of Wood et al. (2005a; e.g. $\dot{M} \propto F_{\mathrm{X}}^{1.34 \pm 0.18}$ ), exploring various possibilities that could explain the discrepancy in these relations.

- Finally, by exploiting the 3D capabilities of our simulations we characterised the stellar wind structure at the inner edge of the HZ of these systems (Sect. 4.2). The optimistic and conservative limits of this boundary, provided in the HZG (Kane \& Gelino 2012), were considered. We included a $10^{\circ}$ range in orbital inclination (e.g. Fig. 12), in order to provide more realistic stellar wind parameters (allowing possible off-the-equator variations), and to capture the region where the majority of exoplanets have been found so far (Han et al. 2014). The results of this characterisation are presented in Table 4, and consider all the magnetohydrodynamic properties of the stellar wind in these systems. Using the solar simulations, reference calculations at the locations of Mercury, Mars, and the Earth are also provided. These results will be used in a future study to perform a dynamical parametrisation of the inner edge of the $\mathrm{HZ}$ in these and other systems, accounting for the effects due to the stellar wind and the high-energy environment of the host star.
Acknowledgements. We would like to thank the referee for their constructive comments which helped to improve the quality of this paper. This work was carried out using the SWMF/BATSRUS tools developed at The University of Michigan Center for Space Environment Modeling (CSEM) and made available through the NASA Community Coordinated Modeling Center (CCMC). We acknowledge the support by the DFG Cluster of Excellence "Origin and Structure of the Universe". We are grateful for the support by A. Krukau through the Computational Center for Particle and Astrophysics (C2PAP).

\section{References}

Alvarado-Gómez, J. D., Hussain, G. A. J., Grunhut, J., et al. 2015, A\&A, 582, A38

Alvarado-Gómez, J. D., Hussain, G. A. J., Cohen, O., et al. 2016, A\&A, 588, A28

Amard, L., Palacios, A., Charbonnel, C., Gallet, F., \& Bouvier, J. 2016, A\&A, 587, A105

Arzoumanian, D., Jardine, M., Donati, J.-F., Morin, J., \& Johnstone, C. 2011, MNRAS, 410, 2472

Aurière, M. 2003, in EAS Publ. Ser. 9, eds. J. Arnaud, \& N. Meunier, 105

Barnes, S. A., \& Kim, Y.-C. 2010, ApJ, 721, 675

Blackman, E. G., \& Owen, J. E. 2016, MNRAS, 458, 1548

Brown, S. F., Donati, J.-F., Rees, D. E., \& Semel, M. 1991, A\&A, 250, 463

Butler, R. P., Wright, J. T., Marcy, G. W., et al. 2006, ApJ, 646, 505

Cohen, O. 2011, MNRAS, 417, 2592

Cohen, O., \& Drake, J. J. 2014, ApJ, 783, 55

Cohen, O., Drake, J. J., Kashyap, V. L., Hussain, G. A. J., \& Gombosi, T. I. 2010, ApJ, 721, 80

Cohen, O., Kashyap, V. L., Drake, J. J., Sokolov, I. V., \& Gombosi, T. I. 2011, ApJ, 738, 166

Cohen, O., Drake, J. J., Glocer, A., et al. 2014, ApJ, 790, 57

Cranmer, S. R., \& Saar, S. H. 2011, ApJ, 741, 54

De Moortel, I., \& Browning, P. 2015, Philosoph. Trans. R. Soc. Lond. Ser. A, 373, 20140269

De Pontieu, B., McIntosh, S. W., Carlsson, M., et al. 2007, Science, 318, 1574

do Nascimento, Jr., J.-D., Vidotto, A. A., Petit, P., et al. 2016, ApJ, 820, L15

Donahue, R. A., Saar, S. H., \& Baliunas, S. L. 1996, ApJ, 466, 384

Donati, J.-F., \& Brown, S. F. 1997, A\&A, 326, 1135

Donati, J.-F., Howarth, I. D., Jardine, M. M., et al. 2006, MNRAS, 370, 629

Drake, J. J., \& Smith, G. 1993, ApJ, 412, 797

Drake, J. J., Peres, G., Orlando, S., Laming, J. M., \& Maggio, A. 2000, ApJ, 545, 1074

Drake, J. J., Cohen, O., Yashiro, S., \& Gopalswamy, N. 2013, ApJ, 764, 170

Drake, J. F., Swisdak, M., \& Opher, M. 2015, ApJ, 808, L44

Farrell, W. M., Desch, M. D., \& Zarka, P. 1999, J. Geophys. Res., 104, 14025

Fisk, L. A., \& Gloeckler, G. 2014, ApJ, 789, 41

Forget, F., \& Leconte, J. 2014, Philosoph. Trans. Roy. Soc. Lond. Ser. A, 372, 30084

Frey, H. U., Phan, T. D., Fuselier, S. A., \& Mende, S. B. 2003, Nature, 426, 533 Gallet, F., \& Bouvier, J. 2013, A\&A, 556, A36

Garraffo, C., Cohen, O., Drake, J. J., \& Downs, C. 2013, ApJ, 764, 32

Garraffo, C., Drake, J. J., \& Cohen, O. 2015a, ApJ, 807, L6

Garraffo, C., Drake, J. J., \& Cohen, O. 2015b, ApJ, 813, 40

George, S. J., \& Stevens, I. R. 2007, MNRAS, 382, 455

Ghezzi, L., Cunha, K., Smith, V. V., et al. 2010, ApJ, 720, 1290

Gloeckler, G., \& Fisk, L. A. 2015, ApJ, 806, L27

Grießmeier, J.-M., Zarka, P., \& Spreeuw, H. 2007, A\&A, 475, 359

Gry, C., \& Jenkins, E. B. 2014, A\&A, 567, A58

Gurnett, D. A., Kurth, W. S., Burlaga, L. F., \& Ness, N. F. 2013, Science, 341, 1489

Hallinan, G., Sirothia, S. K., Antonova, A., et al. 2013, ApJ, 762, 34

Han, E., Wang, S. X., Wright, J. T., et al. 2014, PASP, 126, 827

Hathaway, D. H. 2015, Liv. Rev. Sol. Phys., 12, 4

Hatzes, A. P. 2014, Nature, 513, 353

Hatzes, A. P., Cochran, W. D., McArthur, B., et al. 2000, ApJ, 544, L145

Hussain, G. A. J., Donati, J.-F., Collier Cameron, A., \& Barnes, J. R. 2000, MNRAS, 318, 961

Hussain, G. A. J., Jardine, M., \& Collier Cameron, A. 2001, MNRAS, 322, 681

Hussain, G. A. J., Alvarado-Gómez, J. D., Grunhut, J., et al. 2016, A\&A, 585, A77

Jardine, M., \& Collier Cameron, A. 2008, A\&A, 490, 843

Jeffers, S. V., Petit, P., Marsden, S. C., et al. 2014, A\&A, 569, A79

Johnstone, C. P., Güdel, M., Brott, I., \& Lüftinger, T. 2015a, A\&A, 577, A28 
Johnstone, C. P., Güdel, M., Lüftinger, T., Toth, G., \& Brott, I. 2015b, A\&A, 577, A27

Kane, S. R., \& Gelino, D. M. 2012, PASP, 124, 323

Kay, C., Opher, M., \& Kornbleuth, M. 2016, ApJ, 826, 195

Khodachenko, M. L., Ribas, I., Lammer, H., et al. 2007, Astrobiology, 7, 167

Koen, C., Kilkenny, D., van Wyk, F., \& Marang, F. 2010, MNRAS, 403, 1949

Kopparapu, R. K., Ramirez, R., Kasting, J. F., et al. 2013, ApJ, 765, 131

Kopparapu, R. K., Ramirez, R. M., SchottelKotte, J., et al. 2014, ApJ, 787, L29

Koutroumpa, D., Lallement, R., Raymond, J. C., \& Kharchenko, V. 2009, ApJ, 696,1517

Lammer, H. 2013, Origin and Evolution of Planetary Atmospheres (Berlin Heidelberg: Springer)

Lammer, H., Selsis, F., Ribas, I., et al. 2003, ApJ, 598, L121

Lammer, H., Lichtenegger, H. I. M., Kulikov, Y. N., et al. 2007, Astrobiology, 7, 185

Lang, P., Jardine, M., Morin, J., et al. 2014, MNRAS, 439, 2122

Lazio, T. J. W., \& Farrell, W. M. 2007, ApJ, 668, 1182

Lazio, W. T. J., Farrell, W. M., Dietrick, J., et al. 2004, ApJ, 612, 511

Lazio, T. J. W., Carmichael, S., Clark, J., et al. 2010a, AJ, 139, 96

Lazio, T. J. W., Shankland, P. D., Farrell, W. M., \& Blank, D. L. 2010b, AJ, 140, 1929

Lecavelier Des Etangs, A., Sirothia, S. K., Gopal-Krishna, \& Zarka, P. 2009, A\&A, 500, L51

Lecavelier Des Etangs, A., Sirothia, S. K., Gopal-Krishna, \& Zarka, P. 2011, A\&A, 533, A50

Lecavelier des Etangs, A., Sirothia, S. K., Gopal-Krishna, \& Zarka, P. 2013 A\&A, 552, A65

Linsky, J. L., \& Wood, B. E. 2014, ASTRA Proc., 1, 43

Llama, J., Wood, K., Jardine, M., et al. 2011, MNRAS, 416, L41

Llama, J., Vidotto, A. A., Jardine, M., et al. 2013, MNRAS, 436, 2179

Lundin, R., Lammer, H., \& Ribas, I. 2007, Space Sci. Rev., 129, 245

Malamut, C., Redfield, S., Linsky, J. L., Wood, B. E., \& Ayres, T. R. 2014, ApJ, 787, 75

Matt, S., \& Pudritz, R. E. 2008, ApJ, 678, 1109

Matt, S. P., MacGregor, K. B., Pinsonneault, M. H., \& Greene, T. P. 2012, ApJ, 754, L26

Matt, S. P., Brun, A. S., Baraffe, I., Bouvier, J., \& Chabrier, G. 2015, ApJ, 799, L23

Mayor, M., Udry, S., Naef, D., et al. 2004, A\&A, 415, 391

McComas, D. J., Dayeh, M. A., Funsten, H. O., Livadiotis, G., \& Schwadron, N. A. 2013, ApJ, 771, 77

McIntosh, S. W., de Pontieu, B., Carlsson, M., et al. 2011, Nature, 475, 477

Mignone, A., Bodo, G., Massaglia, S., et al. 2007, ApJS, 170, 228

Naef, D., Mayor, M., Pepe, F., et al. 2001, A\&A, 375, 205

Ness, J.-U., \& Jordan, C. 2008, MNRAS, 385, 1691

Nichols, J. D. 2011, MNRAS, 414, 2125

Nichols, J. D., \& Milan, S. E. 2016, MNRAS, 461, 2353

Nicholson, B. A., Vidotto, A. A., Mengel, M., et al. 2016, MNRAS, 459, 1907

Opher, M., Drake, J. F., Zieger, B., \& Gombosi, T. I. 2015, ApJ, 800, L28

Oran, R., van der Holst, B., Landi, E., et al. 2013, ApJ, 778, 176

Pepe, F., Ehrenreich, D., \& Meyer, M. R. 2014, Nature, 513, 358

Piskunov, N., \& Kochukhov, O. 2002, A\&A, 381, 736

Piskunov, N., Snik, F., Dolgopolov, A., et al. 2011, The Messenger, 143, 7

Powell, K. G., Roe, P. L., Linde, T. J., Gombosi, T. I., \& De Zeeuw, D. L. 1999, J. Comput. Phys., 154, 284

Pulkkinen, T. 2007, Liv. Rev. Sol. Phys., 4, 1

Redfield, S., \& Falcon, R. E. 2008, ApJ, 683, 207
Redfield, S., \& Linsky, J. L. 2004a, ApJ, 602, 776

Redfield, S., \& Linsky, J. L. 2004b, ApJ, 613, 1004

Redfield, S., \& Linsky, J. L. 2008, ApJ, 673, 283

Redfield, S., \& Linsky, J. L. 2015, ApJ, 812, 125

Reiners, A., \& Mohanty, S. 2012, ApJ, 746, 43

Réville, V., Brun, A. S., Matt, S. P., Strugarek, A., \& Pinto, R. F. 2015a, ApJ, 798, 116

Réville, V., Brun, A. S., Strugarek, A., et al. 2015b, ApJ, 814, 99

Sanz-Forcada, J., Micela, G., Ribas, I., et al. 2011, A\&A, 532, A6

Sanz-Forcada, J., Stelzer, B., \& Metcalfe, T. S. 2013, A\&A, 553, L6

Schmitt, J. H. M. M., \& Liefke, C. 2004, A\&A, 417, 651

Semel, M. 1989, A\&A, 225, 456

Shaikhislamov, I. F., Khodachenko, M. L., Sasunov, Y. L., et al. 2014, ApJ, 795, 132

Shkolnik, E., Walker, G. A. H., Bohlender, D. A., Gu, P.-G., \& Kürster, M. 2005, ApJ, 622, 1075

Shkolnik, E., Bohlender, D. A., Walker, G. A. H., \& Collier Cameron, A. 2008, ApJ, 676, 628

Sirothia, S. K., Lecavelier des Etangs, A., Gopal-Krishna, K. N. G., \& Ishwar-Chandra, C. H. 2014, A\&A, 562, A108

Sokolov, I. V., van der Holst, B., Oran, R., et al. 2013, ApJ, 764, 23

Stevens, I. R. 2005, MNRAS, 356, 1053

Strugarek, A., Brun, A. S., Matt, S. P., \& Réville, V. 2014, ApJ, 795, 86

Strugarek, A., Brun, A. S., Matt, S. P., \& Réville, V. 2015, ApJ, 815, 111

Suzuki, T. K., Imada, S., Kataoka, R., et al. 2013, PASJ, 65, 9821

Takeda, G., Ford, E. B., Sills, A., et al. 2007, ApJS, 168, 297

Terada, N., Kulikov, Y. N., Lammer, H., et al. 2009, Astrobiology, 9, 55

Testa, P., Saar, S. H., \& Drake, J. J. 2015, Philosoph. Trans. Roy. Soc. Lond. Ser. A, 373, 20140259

Tóth, G., Sokolov, I. V., Gombosi, T. I., et al. 2005, J. Geophys. Res., 110, A12226

Tóth, G., van der Holst, B., \& Huang, Z. 2011, ApJ, 732, 102

Tóth, G., van der Holst, B., Sokolov, I. V., et al. 2012, J. Comput. Phys., 231, 870

Valenti, J. A., \& Fischer, D. A. 2005, ApJS, 159, 141

van der Holst, B., Sokolov, I. V., Meng, X., et al. 2014, ApJ, 782, 81

Vidotto, A. A., Jardine, M., \& Helling, C. 2010, ApJ, 722, L168

Vidotto, A. A., Fares, R., Jardine, M., et al. 2012, MNRAS, 423, 3285

Vidotto, A. A., Jardine, M., Morin, J., et al. 2014, MNRAS, 438, 1162

Vidotto, A. A., Fares, R., Jardine, M., Moutou, C., \& Donati, J.-F. 2015, MNRAS, 449, 4117

Vidotto, A. A., Donati, J.-F., Jardine, M., et al. 2016, MNRAS, 455, L52

Webb, D. F., \& Howard, T. A. 2012, Liv. Rev. Sol. Phys., 9, 3

Wood, B. E. 2004, Liv. Rev. Sol. Phys., 1, 2

Wood, B. E., Müller, H.-R., Zank, G. P., \& Linsky, J. L. 2002, ApJ, 574, 412

Wood, B. E., Müller, H.-R., Zank, G. P., Linsky, J. L., \& Redfield, S. 2005a, ApJ, 628, L143

Wood, B. E., Redfield, S., Linsky, J. L., Müller, H.-R., \& Zank, G. P. 2005b, ApJS, 159, 118

Wood, B. E., Müller, H.-R., Redfield, S., \& Edelman, E. 2014, ApJ, 781, L33

Wood, B. E., Linsky, J. L., \& Güdel, M. 2015, in Characterizing Stellar and Exoplanetary Environments, eds. H. Lammer, \& M. Khodachenko, Astrophys. Space Sci. Libr., 411, 19

Zarka, P., Treumann, R. A., Ryabov, B. P., \& Ryabov, V. B. 2001, Ap\&SS, 277, 293

Zarka, P., Lazio, J., \& Hallinan, G. 2015, Advancing Astrophysics with the Square Kilometre Array (AASKA14), 120 